\title{
Transport coefficients for shape degrees in terms of Cassini ovaloids *
}

\author{
F.A.Ivanyuk ${ }^{1,2}$, H.Hofmann ${ }^{\dagger 1}$, V.V.Pashkevich ${ }^{3}$ and S.Yamaji ${ }^{4}$ \\ 1) Physik-Department der Technischen Universität München, D-85747 Garching, Germany \\ 2) Institute for Nuclear Research of the Ukrainian Academy of Sciences, Kiev-28, Ukraine \\ 3) Joint Institute for Nuclear Research, 141980 Dubna, Russia \\ 4) Cyclotron Lab., Riken, Wako, Saitama, 351-01, Japan
}

October 7, 2018

\begin{abstract}
Previous computations of the potential landscape with the shapes parameterized in terms of Cassini ovaloids are extended to collective dynamics at finite excitations. Taking fission as the most demanding example of large scale collective motion, transport coefficients are evaluated along a fission path. We concentrate on those for average motion, namely stiffness $C$, friction $\gamma$ and inertia $M$. Their expressions are formulated within a locally harmonic approximation and the help of linear response theory. Different approximations are examined and comparisons are made both with previous studies, which involved different descriptions of single particle dynamics, as well as with macroscopic models. Special attention is paid to an appropriate definition of the deformation of the nuclear density and its relation to that of the single particle potential. For temperatures above $3 \mathrm{MeV}$ the inertia agrees with that of irrotational flow to less than a factor of two, but shows larger deviations below, in particular in its dependence on the shape. Also friction exhibits large fluctuations along the fission path for small excitations. They get smoothed out above $3-4 \mathrm{MeV}$ where $\gamma$ attains values in the range of the wall formula. For $T \geq 2 \mathrm{MeV}$ the inverse relaxation time $\beta=\gamma / M$ turns out to be rather insensitive to the shape and increases with $T$.
\end{abstract}

PACS numbers: 21.60.Ev, 21.60.Cs, 24.10Pa, 24.75+i

to appear in PRC

\section{Introduction}

One of the oldest but still most challenging problems of nuclear physics is an adequate description of collective motion at finite excitations. As the prime example one may

\footnotetext{
* Supported in part by the Deutsche Forschungsgemeinschaft

${ }^{\dagger}$ e-mail: hhofmann@physik.tu-muenchen.de

${ }^{\ddagger}$ www home page: http://www.physik.tu-muenchen.de/tumphy/e/T36/hofmann.htm]
} 
quote nuclear fission which has attracted the attention of both experimentalists as well as theoreticians since its discovery. To date it is still an open question which type of configurations the system undergoes on its way from the potential minimum over the saddle region down to scission. Whereas in the early days those of the compound model were clearly favoured in theoretical pictures, after the discovery of the shell model that of independent particle motion came into fashion more and more. This development was enhanced after computers got fast enough such that Hartree-Fock type computations could be done in every lab.

However, there can be little doubt that this picture fails to describe collective motion at finite excitations where one is compelled almost by experimental evidence that the dynamics shows irreversible behaviour, not only by the very nature of the decay process itself but by the appearance of frictional forces. It is more than questionable that this feature can adequately be met by introducing simple minded collision terms. Decent descriptions of fission in terms of the one body density operator most likely require to consider correlations beyond the independent particle picture, together with non-Markovian effects. This is a difficult problem in itself, not to mention the computational task of solving this equation of motion for the one body density.

For these reasons it may still be interesting and worth while to start from a more phenomenological point of view introducing the shape parameters as collective variables. It is true that in this way again the picture of independent particles will serve as a starting point, in the form of the deformed shell model. However, the latter is simple and flexible enough to allow one considering residual interactions, in one way or other. As we shall see, it may be possible to gain insight into their importance by studying dynamical aspects. Likewise, we may be able to get information on the complexity of the configurations which are to be considered. Such a task becomes more feasible in case collective motion is sufficiently slow. Then one may exploit the quasi-static picture which reduces the complexity of the full problem drastically. Under such circumstances one may actually linearize the problem and treat collective motion locally within a harmonic approximation. In this way one may take advantage of the benefits of linear response theory.

One of the major problems in theories of this type is to find a decent guess for the relevant macroscopic variables, a problem which is familiar almost from all transport theories. For nuclear fission there exists some kind of guiding principle through the liquid drop model. The latter is known to represent the static energy for temperatures above $1-2 \mathrm{MeV}$. Since at these temperatures one expects motion to be strongly damped, it will most likely follow somehow the line of steepest decent. Possible shapes which a fissioning nucleus may assume on its way to scission have been looked for in [1] by minimizing the liquid drop energy. This minimization has been done for some realistic energy density functional under the constraint of fixing a parameter which measures the distance between the evolving fragments. Incidentally, it is the same parameter which we are going to exploit later on in our approach. It so turns out that the shapes found in this way can be approximated fairly well by the Cassini ovaloids introduced to nuclear physics in [2]. Later in [3] a single particle model has been constructed for such a parameterization of shapes, which was based on the Woods-Saxon potential.

In this paper we are going to use this model for computations of transport coefficients, after some suitable modifications which are necessary to incorporate the effects mentioned above. One of our goals will be to study average motion along the fission path for different 
temperatures, as it is reflected in the associated transport coefficients of inertia, friction and local stiffness. In this sense the aim of our present work is similar to the one of [4, where a two center shell model was used. The latter feature renders the previous model simpler on the computational level. On the other hand, the parameterization of the shape by means of Cassini ovaloids opens the possibility of treating more realistic shapes, which are perhaps better suited to describe the later stages of a fission process. Furthermore, it is fair to say that the Woods-Saxon potential may be supposed to resemble more the "true" mean field.

For Cassini ovaloids commonly a few parameters suffice to treat in simple terms a whole variety of realistic shapes including very compact ones as well as strongly deformed ones with a well developed neck, or even those corresponding to separated fragments. In this sense this parameterization may be considered superior to expansions in terms of spherical harmonics (see [5]). In the ideal case one would then be able to compute transport tensors for all the parameters, the collective degrees of freedom, one claims to be relevant. This is a tremendous task and so far has been carried through only for a two dimensional model [6], without utilizing though the full microscopic potential of linear response theory. In this paper we want to restrict ourselves to the one dimensional case. The main reason for that is found, of course, in the simplification one gains by this restriction. However, it may be said that at present most of the applications of macroscopic equations of motion to fission at finite excitation adhere to a similar confinement, see e.g. [7] - [10]. Evidently one then needs to rely on the "right" guess of the fission path. As said before and for arguments given there we presume it to be represented well enough by the line along the valley of the static energy. Possible improvements have to be left for future studies.

\section{$2 \quad$ Deformed shell model}

\subsection{Shape parameterization}

We follow the suggestion put forward in [3], but would like to repeat the most important elements for convenience. The Cassini ovaloids are obtained by rotating the curve

$$
\rho(z, \epsilon)=R_{0}\left[\sqrt{a^{4}+4 \epsilon z^{2} / R_{0}^{2}}-z^{2} / R_{0}^{2}-\epsilon\right]^{1 / 2}
$$

around the $z$-axis, with $z$ and $\rho$ being cylindrical coordinates. The constant $a$ is defined by volume conservation, implying that the family of shapes (2.1) depends only on one deformation parameter $\epsilon$. As is easily recognized from (2.1) the value of $\epsilon=0$ corresponds to a sphere. For $0<\epsilon<0.4$ the form resembles very much that of a spheroid with the ratio of the axes given by

$$
\frac{\text { shorter axes }}{\text { longer axes }}=\frac{1-2 \epsilon / 3}{1+\epsilon / 3}
$$

At $\epsilon \approx 0.5$ a neck appears and at $\epsilon=1.0$ the nucleus separates into two fragments. A few examples of the family (2.1) are shown in Fig.1.

It is possible to describe a more general class of axially symmetric shapes by exploiting an expansion about the surface given by (2.1). We may introduce two new coordinates $R$ and $x$ such that $R=$ const $=R_{0}$ corresponds to the Cassini ovals (2.1). The coordinate $x$ specifies the position of a point on the line given by (2.1), see [3] for details. With these 
two variables at our disposal we may parameterize a new shape. The latter is meant to express the deviation from the ovaloid given by (2.1) by means of an expansion into a series of Legendre polynomials

$$
R(x)=R_{0}\left(1+\sum_{n} \alpha_{n} P_{n}(x)\right)
$$

The full set of collective variables or parameters then includes the coefficients $\alpha_{n}$ in addition to the $\epsilon$ from before.

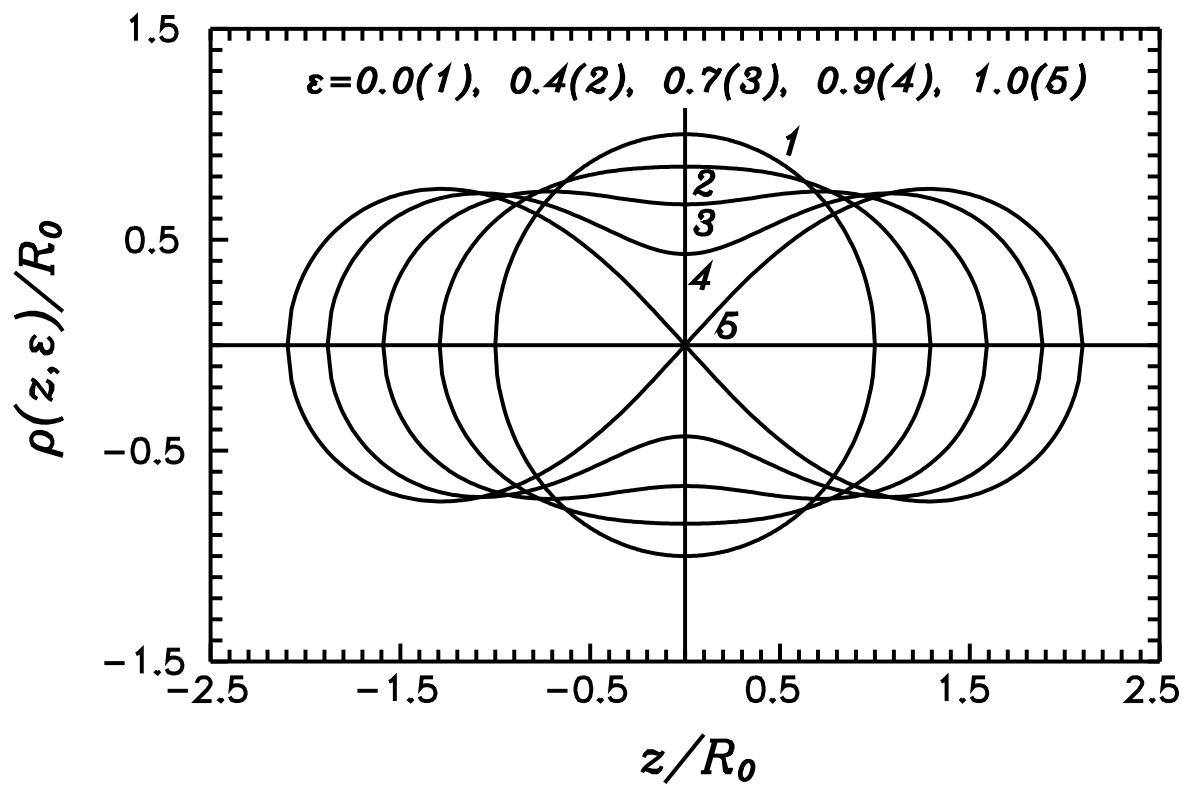

Figure 1: The parameterization of the shape of the nuclear surface in terms of Cassini ovaloids. The values of the deformation parameter $\epsilon$ (see (2.1)) are indicated in the Figure.

Sometimes it is convenient to introduce a measure for the overall elongation of the nucleus instead of $\epsilon$. One may chose, for instance, the distance $R_{12}$ between the left and right center of masses. To have a dimensionless quantity one may divide $R_{12}$ by the diameter $2 R_{0}$ of the sphere (of identical volume) to get

$$
r_{12} \equiv \frac{R_{12}}{2 R_{0}}=\frac{\int\left|z-z_{c m}\right| d V}{R_{0} \int d V}
$$

with $z_{c m}$ being the $z$-coordinate of the center of mass of the the whole complex. The integration is carried out over the volume within the sharp surface specified by (2.3). Asymptotically, the $r_{12}$ turns into half of the distance between centers of mass of the fission fragments. Incidentally, this variable is defined uniquely for any parameterization of the shape and has been used in the past by many authors. This feature facilitates comparisons to theories which are based on shell models with different shape variables. The relation between $r_{12}$ and $\epsilon$ is demonstrated in Fig.2.

\subsection{Single-particle Hamiltonian}

The single-particle Hamiltonian $\hat{h}_{i p m}$ will be constructed like in [3]. It has terms for the kinetic energy $\hat{T}$, the radial potential $\hat{V}$, the spin-orbit coupling $\hat{V}_{\text {s.o. }}$ and the Coulomb 
potential $\hat{V}_{\text {Coul }}$ :

$$
\hat{h}_{\text {ipm }}=\hat{T}+\hat{V}+\hat{V}_{\text {s.o. }}+\hat{V}_{\text {Coul }}
$$

The radial part $\hat{V}$ is represented by a finite depth Woods-Saxon potential

$$
V(\rho, z)=V_{0}[1+\exp (l(\rho, z) / a)]^{-1}
$$

where $l(\rho, z)$ is the shortest distance from the point $(\rho, z)$ to the sharp surface and $a$ is the diffuseness parameter which is assumed to be constant along the surface. The spin-orbit potential may be written in a way which makes apparent that it is proportional to the gradient of the potential given in (2.6),

$$
\hat{V}_{\text {s.o. }} \propto[\vec{s}, \vec{p}] \nabla V
$$

Here $\vec{p}$ and $\vec{s}$ stand for the nucleon's momentum and the spin. The Coulomb potential is calculated for a charge distributed uniformly inside the sharp surface (2.3) or (2.1).

The single-particle energies and wave functions are determined by diagonalizing the matrix of the Hamiltonian (2.5) calculated with the wave functions of a deformed axially symmetric oscillator potential, see [11]. An example of the deformation dependence of the single-particle energies is shown in Fig.3. As the result of diagonalization one obtains not only energies and wave functions of bound states but also those of discrete states of positive energy, which for the Woods-Saxon potential lie in the continuum. The density of these states depends on the number of oscillator shells included into the basis. In the computations within the shell correction method the number of oscillator shells is optimized by the requirement that the states with positive energies provide a smooth extrapolation of the density of bound states into the continuum. Accounting for such states with positive energy improves considerably the "plateau" of the shell correction as a function of the averaging interval. In the present paper we do so not only when calculating deformation energies but also in the computation of transport coefficients. The cut-off in the single-particle energy was set equal to $20 \mathrm{MeV}$. We have checked that a variation of the cut-off energy within the interval $5-20 \mathrm{MeV}$ does not change much the values of the transport coefficients. This may be understood from the fact that these transport coefficients reflect the truly low frequency behavior of the system.

\section{$2.3 \quad$ Deformation energy}

The deformation energy $E_{\text {def }}$ at zero temperature is calculated according to the shell correction method [12], [13] as the sum of the liquid drop energy $E_{\text {def }}^{L D M}$ and the shell correction $\delta E^{n, p}+\delta P^{n, p}$ (including the one for the pairing energy)

$$
E_{d e f}=E_{d e f}^{L D M}+\sum_{p, n}\left(\delta E^{p, n}+\delta P^{p, n}\right)
$$

The liquid drop energy is computed as the contributions from the Coulomb $E_{C o u l}$ and surface $E_{S}$ energies according to [12], [13]

$$
E_{\text {def }}^{L D M}=E_{\text {Coul }}+E_{S}-\left(E_{\text {Coul }}^{0}+E_{S}^{0}\right)
$$

where $E_{C o u l}^{0}$ and $E_{S}^{0}$ are the corresponding energies of the spherical shape. As an example, Fig.4 exhibits the results of the calculation of $E_{d e f}$ and $E_{d e f}^{L D M}$ at zero temperature for ${ }^{224} \mathrm{Th}$ as function of parameters $\epsilon$ and $\alpha_{3}$. 
The temperature dependence of Coulomb and surface energy is accounted for by using the forms

$$
E_{\text {Coul }}(T)=E_{\text {Coul }}(T=0)\left(1-\alpha T^{2}\right), \quad E_{S}(T)=E_{S}(T=0)\left(1-\beta T^{2}\right)
$$

with $\alpha=0.000763 \mathrm{MeV}^{-2}$ and $\beta=0.00553 \mathrm{MeV}^{-2}$ [14. To compute the shell correction at finite temperature we use the phenomenological ansatz proposed in [5]

$$
\delta E^{p, n}(T)+\delta P^{p, n}(T)=\left[\delta E^{p, n}(T=0)+\delta P^{p, n}(T=0)\right] \frac{\tau}{\sinh \tau}
$$

with $\tau=2 \pi^{2} T / \hbar \omega_{0}$ and $\hbar \omega_{0}=41 A^{-1 / 3}$, A For temperatures larger than $1 M e V$ (as considered in the present paper) the shell effects are strongly suppressed. Already at $T=1 \mathrm{MeV}$ the minimum of the total deformation energy almost coincides with the bottom of the liquid drop valley. As said before the latter can be approximated rather well just by the Cassini ovaloids. Thus we restricted our set of deformation parameters to the one parameter $\epsilon$ only, with all the $\alpha_{n}$ put equal to zero. However instead of $\epsilon$ we prefer to use $r_{12}$ defined by 2.4. The $r_{12}$-dependence of the total deformation energy and that of the liquid drop is shown in Fig.5. being the mass number of the fissioning nucleus.

\section{Dynamics in locally harmonic approximation}

In the following we will assume to be given a many body Hamiltonian $\hat{H}\left(\hat{x}_{i}, \hat{p}_{i}, Q(t)\right)$ which depends parametrically on the collective variable $Q$ which specifies the shape of nuclear surface. Although for the computations to be presented below, it will mostly be identical to $r_{12}$, in this section we still prefer to use the general notation $Q$ instead, last but not least to indicate the general validity of the discussion to come. This Hamiltonian is assumed to represent the system's total energy. On the level of the shell model this means to add some c-number terms to the sum over those single particle Hamiltonians introduced in (2.5) (see 15]). As will be discussed in the next section, later on we want to account for collisional damping, which from a principal point of view requires to add a two body interaction $\hat{V}_{\text {res }}^{(2)}\left(\hat{x}_{i}, \hat{p}_{i}\right)$. For the moment it is not very important to know details about the way it will be handled - besides the fact that we claim this interaction to be independent of $Q$.

As a consequence of the latter feature, the generator for collective motion, namely $\partial \hat{H}\left(\hat{x}_{i}, \hat{p}_{i}, Q\right) / \partial Q \equiv \hat{F}\left(\hat{x}_{i}, \hat{p}_{i}, Q\right)$, is of pure one body nature. This operator defines the main source of the coupling between the collective degree of freedom $Q(t)$ and the nucleonic ones. Indeed, within the locally harmonic approximation (LHA) the effective Hamiltonian can be written as

$$
\hat{H}(Q)=\hat{H}\left(Q_{0}\right)+\left(Q-Q_{0}\right) \hat{F}+\frac{1}{2}\left(Q-Q_{0}\right)^{2}\left\langle\frac{\partial^{2} \hat{H}}{\partial Q^{2}}\left(Q_{0}\right)\right\rangle_{Q_{0}, T_{0}}^{\mathrm{qs}}
$$

In the second order term the "nucleonic" part appears only as an average of the corresponding operator. Consistently with the harmonic approximation, this average is to be built with that density operator $\rho_{\mathrm{qs}}\left(Q_{0}\right)$ which in the quasi-static picture is to be calculated with the Hamiltonian at $Q_{0}$, namely $\hat{H}\left(Q_{0}\right)$. It is here where thermal concepts come into play. In this "unperturbed" density operator (for the nucleons) one needs to 
specify the amount of heat the nucleonic (or intrinsic) degrees of freedom have at the given configuration parameterized by $Q_{0}$. The simplest possibility is offered by the canonical distribution $\rho_{\mathrm{qs}}\left(Q_{0}\right) \propto \exp \left(-\hat{H}\left(Q_{0}\right) / T\right)$, to which our computations will be restricted. Clearly this picture has to rely on the assumption of a quick relaxation of the relevant internal degrees of freedom; we will come back to this question later on.

Details about this LHA can be found in many references, see e.g. [16], [17], [18]: There it is also described how this local dynamics can be handled within a suitable application of linear response theory. For this reason, we will only recall the most important theoretical issues.

\subsection{Collective response function}

The local motion in the $Q$-variable can be described in terms of the so called collective response function $\chi_{\text {coll }}(\omega)$. It can be derived by introducing a (hypothetical) external force $\hat{F} \tilde{f}_{\text {ext }}(t)$ and by evaluating how the deviation of $\langle\hat{F}\rangle_{\omega}$ from some properly chosen static value reacts to this external field in linear order:

$$
\delta\langle\hat{F}\rangle_{\omega}=-\chi_{\operatorname{coll}(\omega)} f_{e x t}(\omega)
$$

As shown in [17] and [16] the $\chi_{\text {coll }}(\omega)$ can be brought to the form

$$
\chi_{\operatorname{coll}(\omega)}=\frac{\chi(\omega)}{1+k \chi(\omega)}
$$

Here a response function $\chi(\omega)$ for "intrinsic" motion appears: The $\chi(\omega)$ measures how, at some given shape $Q_{0}$ and for some temperature $T_{0}$, the nucleonic degrees of freedom react to the coupling $\hat{F} \delta Q(\omega)$. Its time dependent version reads

$$
\tilde{\chi}(t-s)=\Theta(t-s) \frac{i}{\hbar} \operatorname{tr}\left(\hat{\rho}_{q s}\left(Q_{0}, T_{0}\right)[\hat{F}(t), \hat{F}(s)]\right) \equiv 2 i \Theta(t-s) \tilde{\chi}^{\prime \prime}(t-s)
$$

In this expression the time development of the field operators is defined by the same Hamiltonian $\hat{H}\left(\hat{x}_{i}, \hat{p}_{i}, Q_{0}\right)$ which appears in the density $\hat{\rho}_{\mathrm{qs}}$. The function $\tilde{\chi}^{\prime \prime}(t-s)$ on the very right stands for the so called dissipative part. In Fourier space the full response separates into real and imaginary parts like $\chi(\omega)=\chi^{\prime}(\omega)+i \chi^{\prime \prime}(\omega)$, the $\chi^{\prime}(\omega)$ sometimes being called reactive part.

As one may guess from the very construction, the derivation of (3.3) relies on quasistatic properties of the nucleonic degrees of freedom. For instance, such properties appear in the coupling constant $k$ which is to be determined from

$$
-k^{-1}=\left(\frac{\partial^{2} E}{\partial Q^{2}}\right)_{S}+\chi(\omega=0) \equiv C(0)+\chi(0)
$$

Moreover, the nucleonic degrees of freedom are assumed to behave ergodic in the sense of having the adiabatic susceptibility $\chi^{\text {ad }}=-\delta\langle\hat{F}\rangle / \delta Q_{S}$ be identical to the isolated one, the static response $\chi(0)$ :

$$
\chi(0)=\chi^{\mathrm{ad}}
$$

\footnotetext{
${ }^{1}$ For a discussion of the general problems of using the concept of temperature for an isolated system see 16 .
} 
As has been demonstrated in [18] this condition is not fulfilled in the deformed shell model, which implies that special measures are to be taken to which we will come to below.

It should be noted that the derivation of (3.3) involves a self-consistency relation between the deformation $Q$ of the mean field and the one of the density. The latter may

be measured by the expectation value $\langle\hat{F}\rangle_{t}$. For linearized dynamics this self-consistency condition reduces to the equation

$$
k\langle\hat{F}\rangle_{t}=Q(t)-Q_{0}
$$

well known from the case of undamped vibrations [5], [15]. Realize, please, that the quantity $\langle\hat{F}\rangle_{t}$ is to be calculated with the actual dynamical nuclear states accounting for their appropriate occupations.

Before concluding this subsection we like to write down a more convenient form for the stiffness $C(0)$ appearing in (3.5). It is defined as the second derivative of the internal energy $E(Q, S)$ with respect to deformation at fixed entropy $S$. Since it is not easy to calculate such a derivative it is better to reexpress it by the one of the free energy $f$ at fixed temperature (see eqs.(A.18)-(A.19) of [17])

$$
C(0)=\left(\frac{\partial^{2} E}{\partial Q^{2}}\right)_{S}=\left(\frac{\partial^{2} f}{\partial Q^{2}}\right)_{T}+\left(\frac{\partial T}{\partial Q}\right)_{S} \frac{\partial^{2} f(Q, T)}{\partial Q \partial T}
$$

In [17] it was found that for temperatures larger than $1.5-2.0 \mathrm{MeV}$ the change of $T$ with the collective coordinate $Q$ is small such that the second term on the right hand side of eq. (3.8) can be neglected. Below we would like to use the shell correction method when calculating static energies. In this method usually the intrinsic energy is involved, rather than the free energy. Thus one needs to relate the derivatives of the free and the intrinsic energy both taken at at fixed temperature. This can be done by differentiating the relation $E=f+T S$ with respect to deformation and obeying that the entropy can be expressed as $S=-(\partial f / \partial T)_{Q}$. As the result one gets

$$
C(0) \approx\left(\frac{\partial^{2} f}{\partial Q^{2}}\right)_{T}=\left(\frac{\partial^{2} E}{\partial Q^{2}}\right)_{T}+T \frac{\partial}{\partial T}\left(\frac{\partial^{2} f}{\partial Q^{2}}\right)_{T} \approx\left(\frac{\partial^{2} E}{\partial Q^{2}}\right)_{T}
$$

Like before, the expression on the very right is justified for larger temperatures, where the change of $\partial^{2} f / \partial Q^{2}$ with $T$ is small. This approximation is used in the computations presented below. In Fig.6 the stiffness (3.9) of the energy (2.8)-(2.11) is shown as function of the deformation parameter $r_{12}$. It is seen that its liquid drop part becomes negative for $r_{12} \geq 0.5$ and the total stiffness exhibits rather strong fluctuations due to shell structure.

\subsection{Transport coefficients}

In general the frequency dependence of $\chi_{\text {coll }}(\omega)$ exhibits a complex structure. Its dissipative part $\chi_{\text {coll }}^{\prime \prime}(\omega)$ represents the strength distribution over all possible modes of the whole nucleus which can be excited by an external force like the one introduced above, namely $\hat{F} \tilde{f}_{\text {ext }}(t)$. Rewriting (3.2) as

$$
\left(\chi_{\operatorname{coll}(\omega)}\right)^{-1} \delta\langle\hat{F}\rangle_{\omega}=-f_{\text {ext }}(\omega)
$$


it follows that the inverse of the collective response function can be interpreted as an integral kernel for the effective equation of motion for the time dependent quantity $\delta\langle\hat{F}\rangle_{t}$. Evidently, this time dependent form of the equation of motion must be expected to contain non-Markovian effects. However, there may be situations for which it becomes possible to reduce this complicated structure to differential form. The clue to this simplification can be found by recalling the case of the damped oscillator for which (3.10) takes on the form

$$
\left(\chi_{\text {osc }}(\omega)\right)^{-1} \delta\langle\hat{F}\rangle_{\omega}=\left(-M^{F} \omega^{2}-\gamma^{F} i \omega+C^{F}\right) \delta\langle\hat{F}\rangle_{\omega}=-f_{\text {ext }}(\omega)
$$

Here the following effective "forces" appear: an inertial one, a friction force and the conservative one which is related to the derivative of the effective collective potential, calculated in linearized form. The associated "transport coefficients" have been marked by an upper index "F", to indicate that they are associated to the quantity $\delta\langle\hat{F}\rangle$.

Now the strategy of how to handle the general case is clear: Whenever there is a pronounced peak in the strength distribution $\chi_{\text {coll }}^{\prime \prime}(\omega)$ we may approximate it by a Lorentzian. Since the latter is defined by three quantities, like the width, the position of the maximum and its height, one may deduce the three transport coefficients which appear in the oscillator response, for instance as given by (3.11). In principle, such a procedure may be applied to any one of the peaks of the original strength distribution, which allows one to deduce these transport coefficients for all the possible collective modes, the low frequency ones as well as the high frequency ones. As a matter of fact, such a scheme is commonly adopted for the description of collective vibrations, as discussed in [5], [15]. The difference to the present application is seen in the fact that we want to apply this procedure to describe global dynamics in locally harmonic fashion. The latter aspect puts an additional constraint. As mentioned earlier, our application of linear response theory goes along with the assumption that collective motion is slow, such that the nucleonic degrees of freedom follow closely a thermal equilibrium. We may recall that in some formulas given above, this equilibrium has been parameterized by the quasi-static density operator $\hat{\rho}_{\mathrm{qs}}(Q, T)$. Estimates of the time scale on which such a relaxation can be expected to occur will be given below. But already on this level of information, it is clear that the whole concept would probably not work if high frequency collective modes would be important. For this reason we are bound to concentrate on low frequency ones, if the construction of the transport coefficients is to be consistent with the basic assumptions for the applicability of the quasi-static picture.

In practice the "fit" of the strength distributions of the oscillator model to the "correct" one involves the full response functions, not only their dissipative parts. In short such an adjustment may be characterized by $\left(\chi_{\text {coll }}(\omega)\right)^{-1} \delta\langle\hat{F}\rangle_{\omega} \simeq\left(\chi_{\text {osc }}(\omega)\right)^{-1} \delta\langle\hat{F}\rangle_{\omega}$. A more correct form can be written as a variational procedure

$$
\delta \int_{0}^{\omega_{\max }}\left|k^{2} \chi_{\mathrm{coll}}(\omega)-\frac{1}{-M\left(\omega_{1}\right) \omega^{2}-i \gamma\left(\omega_{1}\right) \omega+C\left(\omega_{1}\right)}\right|^{2} d \omega=0
$$

with the variation to be performed with respect to the coefficients $C\left(\omega_{1}\right), \gamma\left(\omega_{1}\right)$ and $M\left(\omega_{1}\right)$. In this way both real and imaginary parts of $\chi_{\text {coll }}(\omega)$ are fitted simultaneously. Fortunately, for the practical applications to be discussed below it turns out that the coefficients $C\left(\omega_{1}\right), \gamma\left(\omega_{1}\right)$ and $M\left(\omega_{1}\right)$ are rather insensitive to the upper integration limit $\omega_{\max }$. The value of the latter was fixed to be $\hbar \omega_{\max }=5 \mathrm{MeV}$.

The reader will have noticed the appearance of the factor $k^{2}$ in (3.12), as well as the fact that we have left out the upper index $F$ in the transport coefficients. This change is 
easily understood by referring to the self-consistency condition (3.7). The latter can be interpreted as a transformation from the $F$-mode to the $Q$-mode. It implies a corresponding transformation both for the response functions as well as for the transport coefficients (for details see [16]). In this sense the $k^{2} \chi_{\text {coll }}(\omega)$ and the transport coefficients $\mathcal{T}=M, \gamma, C$ stand for the collective response and the coefficients of the $Q$-mode, respectively, with $\mathcal{T}^{F}=k^{2} \mathcal{T}$.

It may turn out that collective motion is so slow, as compared to the dynamics of the nucleons, that the transport coefficients can be deduced by expanding the response functions around $\omega=0$. At first such a procedure has been applied to the intrinsic response (cf.[19]). In this way one may get an approximate solution of the secular equation for the position of the poles of the collective response (3.3). One may write

$$
\frac{1}{k}+\chi(\omega) \approx\left(\frac{1}{k}+\chi(0)\right)+\omega\left(\frac{\partial \chi}{\partial \omega}\right)_{\omega=0}+\omega^{2}\left(\frac{1}{2} \frac{\partial^{2} \chi}{\partial \omega^{2}}\right)_{\omega=0}=0
$$

This form invites to define coefficients for friction $\gamma(0)$ and inertia $M(0)$ by

$$
\gamma(0)=-\left.i \frac{\partial \chi(\omega)}{\partial \omega}\right|_{\omega=0}=\left.\frac{\partial \chi^{\prime \prime}(\omega)}{\partial \omega}\right|_{\omega=0}
$$

and

$$
M(0)=\left.\frac{1}{2} \frac{\partial^{2} \chi(\omega)}{\partial \omega^{2}}\right|_{\omega=0}=\left.\frac{1}{2} \frac{\partial^{2} \chi^{\prime}(\omega)}{\partial \omega^{2}}\right|_{\omega=0}
$$

which in the past have been called the coefficients in "zero frequency" limit. The effective stiffness $C(0)$ is seen to be identical to the local stiffness of the quasi-static energy (mind (3.5)), as one would expect to hold true for slow motion, indeed. Incidentally, the expression for the inertia can be shown to be a generalization of the one of the cranking model to the case of damped motion [19]. Unfortunately, for strong damping this $M(0)$ becomes very small, and sometimes even negative. For practical applications this does not always lead to problems, as usually damping may be so strong that inertia must drop out of the macroscopic equations of motion. Nevertheless, this behaviour of the inertia $M(0)$ is very unpleasant, but fortunately, one can do better.

Rather than to concentrate just on the denominator of (3.3), it is better to take into account the full information contained in this expression. As we may recall this was done implicitly when constructing the transport coefficients $M\left(\omega_{1}\right), \gamma\left(\omega_{1}\right)$ and $C\left(\omega_{1}\right)$ by approximating (3.10) by (3.11). An equation like (3.11) may be obtained by expanding $\left(\chi_{\text {coll }}(\omega)\right)^{-1}$ in $(\overline{3.10})$ to second order in $\omega$. In this way one gets

$$
\begin{gathered}
\left.C \approx \frac{1}{k^{2} \chi_{\operatorname{coll}(\omega)}}\right|_{\omega=0}=\frac{\chi(0)+C(0)}{\chi(0)} C(0) \\
\left.\gamma \approx \frac{1}{k^{2}} \frac{\partial\left(\chi_{\operatorname{coll}(\omega)}\right)^{-1}}{\partial \omega}\right|_{\omega=0}=\frac{(\chi(0)+C(0))^{2}}{\chi^{2}(0)} \gamma(0)
\end{gathered}
$$

and

$$
\left.M \approx \frac{1}{2 k^{2}} \frac{\partial^{2}\left(\chi_{\operatorname{coll}(\omega)}\right)^{-1}}{\partial \omega^{2}}\right|_{\omega=0}=\frac{(\chi(0)+C(0))^{2}}{\chi^{2}(0)}\left(M(0)+\frac{\gamma^{2}(0)}{\chi(0)}\right)
$$

As compared to the zero frequency limit defined above, there are two modifications: Whereas all three coefficients obtain a factor of proportionality, only the inertia gets an 
additional contribution. In most practical applications this proportionality factor is not very important, as usually the static response is much larger than the static stiffness: $\chi(0) \gg|C(0)|$. It is only for small excitations and for deformed oscillator shell models, in particular, that the size of $|C(0)|$ becomes comparable to that of $\chi(0)$. However, the additional term in (3.18) ensures that the modified inertia does not drop indefinitely anymore with increasing damping. Later on we will demonstrate with the help of numerical results that (3.17) and (3.18) approximate the selfconsistent friction $\gamma\left(\omega_{1}\right)$ and inertia $M\left(\omega_{1}\right)$ very well at temperatures $T \geq 2 \mathrm{MeV}$. To distinguish from the zero frequency limit (3.14) and (3.15) we will associate the approximation (3.17) and (3.18) to "the zero frequency limit for the collective response function".

Finally, we should like to mention that relations similar to the ones given in (3.16)(3.18) were obtained earlier in 20], namely for the model case that the collective response function just consists of one (approximately Lorentzian) peak. Solving the equations for $C(0), \gamma(0)$ and $M(0)$ given in [20] with respect to $C, \gamma$ and $M$ one gets back to the equations (3.16)-(3.18).

\subsection{Transformation to sharp densities}

In the previous section we found microscopic expressions of transport coefficients for large scale motion. For many reasons it is desirable to compare them with those of "macroscopic models" [21], like there are the liquid drop model for the inertia and the wall formula for dissipation - not to mention stiffness, which we have seen to become identical to the one of the static energy anyway, as soon as collective motion becomes sufficiently slow. In 18 many points of principle nature have been clarified about how the macroscopic limit can be obtained in microscopic theories, concentrating largely on vibrations around stable configurations. Here we like to look at another, more practical, albeit very important issue.

By its very nature, these macroscopic models assume the nuclear density to be constant inside some surface at which the density drops from the nuclear matter value down to zero within zero range. Commonly this surface is parameterized with the same set of shape variables which in our description define the deformation of the mean field, for which in the present discussion we have chosen the one collective coordinate $Q$. Contrary to the macroscopic picture, the microscopic calculation leads to a density distribution with a soft surface, which in addition depends essentially on the occupation of states. Take the simple case of a spherical potential: if a shell with fixed angular momentum $l$ is not filled completely the corresponding density distribution will not be spherically symmetric. On the other hand, it is clear that the transport coefficients $\mathcal{T}$ will depend sensibly on the distribution of matter. Thus one needs to employ some specific transformation to relate them from one case to the other. This is best done looking at the mean value (or moment) of the operator $\hat{F}$, as a representative for the average density.

In the case of our microscopic picture, we have seen the self-consistency condition (3.7) to imply the relation $\mathcal{T}^{F}=k^{2} \mathcal{T}$ between the transport coefficients of the $F$ and $Q$-mode. All we need to do is to search for a similar condition which translates the $F$-motion into that of the sharp density distribution, with the latter being expressed through the corresponding shape parameter. Such a relation can be found by applying the hypothesis, which we will substantiate below, that the average $\langle F\rangle_{\text {sharp }}$, calculated with a density distribution having a sharp surface, can be approximated well by applying 
an appropriate Strutinsky smoothing to the shell model density. The latter is defined as

$$
\langle F\rangle_{\text {sharp }} \approx \sum_{j} F_{j j} \tilde{n}_{j}
$$

where the $\tilde{n}_{j}$ are smoothed occupation numbers [12], [13]. How they may be used not only to calculate static expectation values like in (3.19), but of corresponding response functions as well has been studied in 222 and [18. The desired relation between $\langle F\rangle_{\text {sharp }}$ and $Q$ may now be obtained simply by applying to (3.19) the derivative with respect to $Q$. One finds

$$
\frac{\partial\langle\hat{F}\rangle_{\text {sharp }}}{\partial Q} \approx \frac{\partial}{\partial Q} \sum_{j} F_{j j} \tilde{n}_{j}=\sum_{j k} \frac{\tilde{n}_{j}-\tilde{n}_{k}}{\epsilon_{j}-\epsilon_{k}}\left|F_{j k}\right|^{2}+\sum_{j} \frac{\partial \tilde{n}_{j}}{\partial Q} \frac{\partial \epsilon_{j}}{\partial Q} \equiv-\chi^{\gamma}
$$

which may be used to deduce $\mathcal{T}_{\text {sharp }}=\left(\chi^{\gamma}\right)^{2} \mathcal{T}_{\text {sharp }}^{F}$. Here, $\mathcal{T}_{\text {sharp }}$ represents the transport coefficients for the sharp density distribution, but calculated for the associated $Q$-mode. Combining this relation with the previous one, we get

$$
\mathcal{T}_{\text {sharp }}=\left(\chi^{\gamma}\right)^{2} \mathcal{T}_{\text {sharp }}^{F} \approx\left(\chi^{\gamma}\right)^{2} \mathcal{T}^{F}\left(\omega_{1}\right)=\left(k \chi^{\gamma}\right)^{2} \mathcal{T}\left(\omega_{1}\right)
$$

As the only one further approximation we have assumed that both density distributions lead to the same averaged value of the field operator $\hat{F}$. In (3.21) there appear on the very left and on the very right the transport coefficients for the $Q$-mode, once for the sharp surface of the macroscopic models and once for the collective coordinate specifying the mean field. We may add here that the quantity $\chi^{\gamma}$ has a physical meaning similar to that of a static susceptibility, hence the choice of this symbol. The only difference to the isothermal susceptibility is found in using the smoothed occupation numbers $\tilde{n}_{j}\left(e_{j}\right)$ instead of $n_{j}\left(e_{j} ; T\right)$.

Let us turn now to "prove" the hypothesis made. This can be done explicitly for the simple case of the deformed oscillator potential. For the more general case we want to appeal to physical intuition, to the extent of accepting the idea that by its very construction Strutinsky smoothing commonly does lead to the macroscopic picture.

Suppose we are given a spheroid whose deformation $Q$ is fixed by the ratio between the semi-axes in $z$-direction and the one perpendicular to it: $Q=z_{0} / y_{0}=\omega_{\perp} / \omega_{z}$ The sharp surface $S$ is then given by

$$
\frac{x^{2}+y^{2}}{y_{0}^{2}}+\frac{z^{2}}{z_{0}^{2}}=1
$$

The deformation of the sharp density may be classified by the average of the quadrupole operator $\hat{Q}_{2}(\vec{r})=2 z^{2}-x^{2}-y^{2}$ calculated as

$$
\left\langle Q_{2}\right\rangle_{\text {sharp }}=\int Q_{2}(\vec{r}) \rho_{0}(\vec{r}) d \vec{r}=\frac{2 A}{5}\left(z_{0}^{2}-y_{0}^{2}\right)
$$

Here, $\rho_{0}(\vec{r})$ measures the density of homo- geneous nuclear matter representing $A$ nucleons distributed uniformly within this surface. In Fig.7 the quadrupole moment (3.23) is plotted by the line with stars as function of the deformation parameter $Q=\omega_{\perp} / \omega_{z}$.

Conversely, the surface (3.22) may be interpreted as an equipotential surface of the corresponding deformed oscillator potential \&. For such a potential one may compute

\footnotetext{
${ }^{2}$ For this case the quadrupole operator $\hat{Q}_{2}$ would be related to our field operator $\hat{F}$ taken at spherical shape by $\left.F\right|_{Q=1}=-\frac{1}{3} m \omega_{0}^{2} \hat{Q}_{2}$.
} 
single-particle wave functions $\varphi_{i}(\vec{r})$ and from them the moment of the microscopic density as

$$
\left\langle Q_{2}\right\rangle_{\text {dens }}=\int Q_{2}(\vec{r}) \sum_{j} n_{j}\left|\varphi_{j}(\vec{r})\right|^{2} d \vec{r}
$$

Obviously this moment $\left\langle Q_{2}\right\rangle_{\text {dens }}$ depends on the occupation numbers $n_{j}$. In Fig.7 we show curves corresponding to three choices of $n_{j}$ : Dotted line: the occupation numbers are fixed at the spherical shape, where the lowest energy states are filled, and kept constant, independent of $Q$ ("diabatic" situation); dashed curve: at each crossing of states the particles and holes are redistributed in such a way that always the lowest states are occupied ("adiabatic" situation); solid curve: here, the smoothed occupation numbers of the shell correction method are used. We see that for the diabatic occupation numbers the quadrupole moment (3.24) differs substantially from that of the sharp density distribution $\left\langle Q_{2}\right\rangle_{\text {sharp }}$ (line with stars), approximately by a factor of two. Recall that this sharp density distribution reflects the deformation of the potential.

On the contrary, the density computed with the adiabatic occupation numbers follows the deformation of the potential on average. It can be said that this feature is one of the basic elements of the Copenhagen picture of collective motion [5] (see also [15]). Indeed, such a redistribution of particles leads to a consistency between the shapes of the potential and the density. For static situations, this leads to the well known relation between the occupation numbers in the various directions and the corresponding frequencies of the potential. In the treatment of [0] this relation is fulfilled for specific deformations. In Fig.7, the latter correspond to the points where the broken line crosses that with the stars. For this broken line it is hardly possible to define precisely the derivative of $\left\langle Q_{2}\right\rangle_{\text {dens }}$ with respect to the deformation parameter. On the other hand, the condition just mentioned can be fulfilled everywhere using the Strutinsky smoothed occupation numbers. In this way the derivative is well defined. Furthermore, the quadrupole moment of the density computed with the smoothed occupation numbers practically coincides with that of the sharp surface distribution.

\section{Microscopic input}

In this section we are going to specify further details of our treatment of nucleonic dynamics. It has already been mentioned that the mere shell model is not sufficient. At finite excitations the effects of collisions cannot be neglected; one even expects them to become the more important the higher the nucleonic temperature will be. To treat collisions on the basis of an explicit form of a two body interaction $\hat{V}_{r e s}^{(2)}\left(\hat{x}_{i}, \hat{p}_{i}\right)$ is hardly possible. Therefore we follow another path and parameterize the effect it would have on the single particle energies. Details of this method can be found in the publications mentioned before, in particular in [16] (see also [23], 24], 18]).

\subsection{Intrinsic response function}

The Fourier transform of the intrinsic response function given in (3.4) can be expressed as the sum over single-particle states

$$
\chi(\omega)=\sum_{j k} \chi_{j k}(\omega)\left|F_{j k}\right|^{2}
$$


with

$$
\chi_{j k}(\omega)=-\int_{-\infty}^{\infty} \frac{d \Omega}{2 \pi \hbar} n(\Omega)\left(\varrho_{k}(\Omega) \mathcal{G}_{j}(\Omega+\omega+i \epsilon)+\varrho_{j}(\Omega) \mathcal{G}_{k}(\Omega-\omega-i \epsilon)\right)
$$

Here $n(x)$ is the Fermi function determining the occupation of single-particle levels. The $\varrho_{k}(\omega)$ represents the distribution of single-particle strength over more complicated states. It may be parameterized by

$$
\varrho_{k}(\omega)=\frac{\Gamma(\omega)}{\left(\hbar \omega-e_{k}-\Sigma^{\prime}(\omega)\right)^{2}+\left(\frac{\Gamma(\omega)}{2}\right)^{2}}
$$

in terms of the real and imaginary part of the self-energy $\Sigma(\omega, T)=\Sigma^{\prime}(\omega, T)-i \Gamma(\omega, T) / 2$ which are assumed to have the following forms:

$$
\Gamma(\omega, T)=\frac{1}{\Gamma_{0}} \frac{(\hbar \omega-\mu)^{2}+\pi^{2} T^{2}}{1+\frac{1}{c^{2}}\left[(\hbar \omega-\mu)^{2}+\pi^{2} T^{2}\right]}
$$

and

$$
\Sigma^{\prime}(\omega, T)=\frac{-c^{2}}{2 \Gamma_{0} \sqrt{c^{2}+\pi^{2} T^{2}}} \frac{(\hbar \omega-\mu)}{1+\left[(\hbar \omega-\mu)^{2}+\pi^{2} T^{2}\right] / c^{2}}
$$

Both are connected to each other by a Kramers-Kronig relation. The $\mu$ in (4.4)-(4.5) is the chemical potential and the cut-off parameter $c$ accounts for the fact that the imaginary part of the self-energy does not increase indefinitely when the excitations get away from the Fermi energy. In the present calculation we choose $\Gamma_{0}=33 \mathrm{MeV}$ and $c=20 \mathrm{MeV}$. The $\mathcal{G}_{k}$ appearing in (4.2) is the one-body Green function

$$
\mathcal{G}_{k}(\omega \pm i \epsilon)=\frac{1}{\hbar \omega-\epsilon_{k}-\Sigma^{\prime}(\omega, T) \pm i \Gamma(\omega, T) / 2}
$$

which is related to the spectral density $\varrho_{k}$ by

$$
\varrho_{k}(\omega)=i\left(\mathcal{G}_{k}(\omega+i \epsilon)-\mathcal{G}_{k}(\omega-i \epsilon)\right)
$$

Details about the evaluation of the integral in (4.2) are given in the Appendix.

For future purpose we want to use this form (4.2) of the response function and write down a more detailed expression for the friction coefficient in zero frequency limit:

$$
\gamma(0)=-\int \frac{d \hbar \Omega}{4 \pi} \frac{\partial n(\Omega)}{\partial \Omega} \sum_{j k}\left|F_{j k}\right|^{2} \varrho_{k}(\Omega) \varrho_{j}(\Omega)
$$

It is obtained (see [18) by substituting eqs. (4.1) - (4.2) into (3.14).

\subsection{The problem of ergodicity}

In section 3.1 it was mentioned that the condition of ergodicity (3.6) is hard to fulfill in the deformed shell model. This statement refers to the study presented in [18], where it was shown that even collisional damping does not help, at least in the version as used to date. One important reason was seen to lie in the fact that our renormalized single particle energies have the same degeneracies as those of the pure shell model. But these 
degeneracies are by far bigger and happen much more often than one would expect for configurations of the compound nucleus. If one believes the latter to be important which should be the case for a fissioning system which has to overcome a large barrier one needs to take special measures to cure the problem just mentioned.

One way to make the deficiencies apparent is to look at the heat pole which shows up either in the correlation function $\psi^{\prime \prime}(\omega)$, or in the relaxation function $\Phi^{\prime \prime}(\omega)$. Both are related to the dissipative response as

$$
\chi^{\prime \prime}(\omega)=\frac{1}{\hbar} \tanh \left(\frac{\hbar \omega}{2 T}\right) \psi^{\prime \prime}(\omega) \quad \Phi^{\prime \prime}(\omega)=\chi^{\prime \prime}(\omega) / \omega
$$

(see [18] or 16] for more details as well as for references to the original literature). Both functions have a peak at $\omega=0$, whose width $\Gamma_{T}$ was seen to be twice the single particle width (4.4) calculated at the chemical potential, i.e. $\Gamma_{T}=2 \Gamma(\omega=\mu, T)$. On very general grounds, the height of this peak can be seen to be proportional to the difference of isothermal and isolated susceptibilities, $\chi^{\mathrm{T}}-\chi(0)$. Numerical calculations in [18] showed this height to be large. Rewriting this difference as $\left(\chi^{\mathrm{T}}-\chi^{\mathrm{ad}}\right)+\left(\chi^{\mathrm{ad}}-\chi(0)\right)$ and recalling that in the nuclear case the difference between the isothermal and adiabatic susceptibility, $\left(\chi^{\mathrm{T}}-\chi^{\mathrm{ad}}\right)$, is small [17], [18, the origin is identified to come from a large violation of ergodicity: $\chi^{\text {ad }} \neq \chi(0)$.

This discussion indicates what we can do to cure this problem: Cut the contributions of the heat pole to all functions mentioned previously down to the magnitude it would have in case the system were ergodic. This means to reduce the height of this peak at $\omega=0$ by the factor $\left(\chi^{\mathrm{T}}-\chi^{\mathrm{ad}}\right) /\left(\chi^{\text {ad }}-\chi(0)\right)$. In 18 a system was studied were $\chi^{\mathrm{T}}-\chi^{\text {ad }}$ vanishes identically, such that the reduction of the heat pole amounted to neglect contributions from all states having the same energy. Such a correction can easily be done. In expressions like (4.1) one simply has to restrict the summations in a proper way. Here we like to adopt a similar procedure, in the sense of neglecting the influence of a finite difference $\chi^{\mathrm{T}}-\chi^{\text {ad }}$. At the temperatures considered it will be very small, indeed. On the other hand we go one step further and neglect also contributions from neighboring states whose energy is finite but smaller than the collisional width the particles, which as we just saw also reflects the width of the heat pole.

The consequences of such a manipulation are shown in Fig.8 for the intrinsic response (left part), the corresponding relaxation function $\Phi^{\prime \prime}(\omega)$ (upper right part) and the collective strength distribution $\chi_{\text {coll }}^{\prime \prime}(\omega)$ (lower right part). All of them have been computed for $T=1 \mathrm{MeV}$. The fully drawn lines correspond to calculations where all matrix elements are taken into account. For the dashed curves matrix elements $F_{j k}$ between states of energy difference $\left|\left(e_{k}-e_{j}\right)\right| \leq \Gamma(\mu, T)$ have been discarded. Their contribution to the relaxation function is exhibited in the upper right part by the dotted line. This plot demonstrates nicely that the heat pole can be associated to a Lorentzian of width $\Gamma_{T}$ around $\omega=0$, and thus corresponds to a pole on the imaginary axes [18], [16]. It is interesting to note that for the present calculation $99 \%$ of this Lorentzian is made out only by two pseudo-crossings of single-particle states which takes place close to the Fermi energy. By pseudo-crossing we mean a situation where as function of $Q$ two levels come close but never cross; one such event is encircled in Fig.3. The big contribution to friction in the zero frequency limit of (3.14) (or (3.17)) which results from this heat pole can be estimated looking at the upper right part of Fig.8: The slope of the fully drawn line is much bigger than the one of the dashed line. On the other hand, contributions to $\gamma(0)$ 
from real crossings of levels is very small. This is due to the fact that the matrix elements $\left|F_{j k}\right|$ vanish exactly at the crossing points and are very small in the vicinity of such a (real) crossing.

The influence of the heat pole (in the intrinsic system) on the collective strength distribution can be inferred from the lower right part of Fig.8. The fully drawn line shows a small peak at very small frequencies, say $\hbar \omega \approx 0.2-0.3 \mathrm{MeV}$. Compared to the much bigger strength found in the peak at about $1.5-2 \mathrm{MeV}$ one is inclined to just "forget" the small peak when one wants to define the transport coefficients. Indeed, it somehow looks very natural to associate the bigger peak to the genuine low frequency mode. This may be understood as another argument to leave out the contribution of the heat pole to the transport coefficients, besides the one involving ergodicity for intrinsic motion. All computations to be reported below were done along this line, i.e. contributions to response function from the states with the energy difference $\left|\left(e_{k}-e_{j}\right)\right| \leq \Gamma(\mu, T)$ were not taken into account.

\subsection{The influence of collisional damping on nucleonic relaxation}

The whole formulation of our theory is based on the assumption that the nucleonic degrees of freedom stay close to a thermal equilibrium. The latter is not fixed, however; rather it continuously gets disturbed by collective motion itself, forgetting for the moment a possible evaporation of light particles or gammas. It should thus be of interest to have some estimate of an appropriate relaxation time. The best candidate for this is offered by the "generator" of collective motion, namely the $\hat{F}\left(\hat{x}_{i}, \hat{p}_{i}, Q_{0}\right)$, which defines the coupling of the collective variable to the nucleons. It is predominately this quantity which "decides" which kind of modes of the intrinsic degrees of freedom get excited. We may recall from the discussion in sect. 3 the close relation of this $\hat{F}$ to the nucleonic response function appearing in our theory: The $\chi(\omega)$ parameterizes that average "excitation" $\delta\langle\hat{F}\rangle_{\omega}$ which comes about through a change of the collective variable $\delta Q$. If we are just interested to estimate the time $\tau$ after which the $\delta\langle\hat{F}\rangle_{t}$ has decayed to its static value, we may study the time dependent function $\tilde{\chi}(t)$ given in (3.4). In literal sense it represents the $\delta\langle\hat{F}\rangle_{t}$ if excited by a sharp pulse like $\delta Q(t) \propto \delta(t)$. Notice please, that for $t>0$ the $\tilde{\chi}(t)$ is proportional to the derivative of the Fourier transformed relaxation function, namely $\tilde{\chi}(t) \propto d \tilde{\Phi}^{\prime \prime}(t) / d t$ (see (3.4) and (4.9)). Thus the information contained in $\tilde{\chi}(t)$ is equaivalent to that of $\Phi^{\prime \prime}(t)$ up to an additive constant. The latter measures the long time limit of $\Phi^{\prime \prime}(t)$; it is related to the strength of the heat pole. At the moment we are interested only in the behaviour for finite times.

In Fig.9. results of two different computations of $\tilde{\chi}(t)$ are shown. One case just refers to the deformed shell model, for the other one collisional damping is taken into account. It is clearly seen that only by way of such collisions we may speak of genuine relaxation. It is also observed that the latter does not depend much on the shape. However, the relaxation time $\tau$ decreases considerably with increasing temperature. The latter effect is expected of course from the very form by which the temperature appears in the single particle widths (4.4). From the fully drawn lines of Fig.9 one may deduce for $\tau$ values like $0.5,0.3,0.1 \hbar / \mathrm{MeV}$ for $T=1,2,5 \mathrm{MeV}$. (This may for instance be done by approximating the envelope of $\tilde{\chi}(t)$ by an expontial such that $\tau$ may be defined through the "width" at half maximum.) Later on in sect.5.3 we are going to compare them with typical time scales of collective motion, but we may say already here that this microscopic 
time $\tau$ is at least one order of magnitude smaller than the one which measures motion in the collective variable $Q$.

Finally, we should like to mention that the results found from the present computation are in accord with those reported in [25].

\section{Numerical results for collective transport coeffi- cients}

In this chapter we will discuss the numerical results for transport coefficients $\mathcal{T}$ computed along the fission path of ${ }^{224} \mathrm{Th}$. As it was already mentioned, for temperatures above $1 \mathrm{MeV}$ the fission path is parameterized in terms of Cassini ovaloids by only one deformation parameter $\epsilon$. Since we prefer to use the parameter $r_{12}$ instead of $\epsilon$ we have to relate the transport coefficients accordingly. This can be done by exploiting the following relation, obeying that the $\alpha_{n}$ 's are fixed,

$$
\mathcal{T}_{r_{12} r_{12}}=\mathcal{T}_{\epsilon \epsilon}\left(\frac{d r_{12}}{d \epsilon}\right)^{-2}
$$

which simply follows from general properties of coordinate transformations. The derivative $\partial r_{12} / \partial \epsilon$ is obtained by differentiating (2.4), the result is shown by dashed line in Fig.2. Recall please, that the deformation dependence of the transport coefficients is defined essentially by the choice of the collective variables. For example, $\mathcal{T}_{r_{12} r_{12}}$ decreases with $r_{12}$ but $\mathcal{T}_{\epsilon \epsilon}$ is increases as a function of $\epsilon$. Both in the figures as well as in the text below we will omit the indices $r_{12} r_{12}$, keeping in mind that the transport coefficients are defined with respect to $r_{12}$ (even if sometimes they will be shown as function of $\epsilon$ ).

\subsection{Accuracy of zero frequency limit for the collective response function}

The friction coefficient $\gamma\left(\omega_{1}\right)$ and mass parameter $M\left(\omega_{1}\right)$ defined according to (3.12) are shown in Fig.10 by solid lines as function of the deformation parameter $r_{12}$ for temperatures between 1 and $3 \mathrm{MeV}$. They are compared with calculations for which the approximation (3.17)-(3.18) is used for friction and inertia, respectively. The latter results are marked by dashed curves. As can be seen, this approximation is quite accurate for $T \geq 2 \mathrm{MeV}$. This implies that for such temperatures one may avoid the time consuming computation of the frequency dependence of collective response function. One may compute friction and inertia directly from (3.17) and (3.18). As for the big fluctuations seen at $T=1 \mathrm{MeV}$ we expect them to become much smaller as soon as pairing correlations will be taken into account, which shall be subject of future studies.

For comparison we also show in Fig.10 (by the lines with stars) wall friction $\gamma_{\mathrm{w} . f}$. and the inertia of irrotational flow. According to [26] wall friction $\gamma_{\mathrm{w} . f \text {. is proportional }}$ to the squared normal velocity $u_{n}^{2}(s)$ of the surface, integrated over the nuclear surface. Following [26] this may be deduced from the loss of (collective) energy which is given by

$$
\dot{E}=\frac{3}{4} \rho v_{F} \oint u_{n}^{2}(s) d s=\gamma_{\text {w.f. }} \dot{r}_{12}^{2}(t)
$$


where $\rho$ and $v_{F}$ are the nucleons' density and Fermi velocity. For axially symmetric shapes the surface velocity $u_{n}(s)$ can be expressed in terms of the profile function $\rho(z, \epsilon)$ from eq. (2.1) as

$$
u_{n}(z)=\bar{u}_{n}(z) \dot{r}_{12}(t), \quad \bar{u}_{n}(z)=\frac{1}{\Lambda(z)} \frac{\partial \rho(z, \epsilon)}{\partial \epsilon}\left(\frac{\partial r_{12}}{\partial \epsilon}\right)^{-1}, \quad \Lambda(z)=\sqrt{1+\left(\frac{\partial \rho(z, \epsilon)}{\partial z}\right)^{2}}
$$

The mass parameter of an incompressible irrotational fluid has been computed as suggested in [27]. It can be written as

$$
M_{i r r}=m \oint \xi(s) \bar{u}_{n}(s) d s
$$

with the potential $\xi(\vec{r})$ for the velocity field expressed by the potential of some "surface charge" distribution

$$
\xi(\vec{r})=\frac{1}{2 \pi} \oint \frac{\nu\left(s^{\prime}\right)}{\left|\vec{r}-\vec{r}\left(s^{\prime}\right)\right|} d s^{\prime}
$$

The substitution of (5.5) into the Neumann equation

$$
\Delta \xi=0, \quad(\vec{n} \nabla \xi)_{S}=u_{n}(s)
$$

leads to some integral equation for the density of the "surface charge" $\nu(s)$ which was solved iteratively starting with

$$
\nu^{0}(s)=-\bar{u}_{n}(s)
$$

as zeroth approximation to $\nu(s)$, for details see [27]. We have checked that for the particular case of the shape family (2.1) the Werner-Wheeler method [28] turns out to be a very accurate approximation to the mass parameter (5.4). Both results coincide within the thickness of the lines in the Figure.

\section{$5.2 \quad$ Temperature and deformation dependence of friction and inertia}

In Figs.11 and 12 the friction coefficient and the mass parameter are presented as function of the deformation parameter $\epsilon$ for five different temperatures $T=1-5 \mathrm{MeV}$. They have been calculated from the oscillator fit (3.12), but transformed to sharp densities according to (3.21). The parameter $\epsilon$ is chosen for reasons to be given below. In both figures results of the commonly adopted macroscopic models, namely $\gamma_{w . f}$ and $M_{i r r}$ are shown by the lines with stars. Several observations can be made.

- To some extent the $\gamma_{w . f}$. and $M_{i r r}$ can be said to be reached at the higher temperatures. The very fact that this statement is more true for the inertia but less so for friction can be understood as follows. As shown in [18], $\gamma_{w . f}$. may be considered the macroscopic limit of our model only if such subtleties as collisional damping are left out. Conversely, the high temperature limit of the inertia is related to the value of the energy weighted sum, and the latter is known to be associated to $M_{i r r}$. This is true at least when one treats vibrations within simple models (see [5], [15] for the situations of $T=0$ and [30] at $T \neq 0$ ).

- As has been demonstrated in [4], in the general case the evaluation of the sum rule value becomes somewhat delicate for collisional damping. The nice feature of reaching the $M_{i r r}$ automatically can be considered a proof of the usefulness of the transformation (3.21). 
- Like in the calculations of [4], on average friction is seen to increase with $T$ whereas the inertia decreases.

- Again similarly to [4], both transport coefficients decrease with deformation on average. - The coordinate dependence shows fluctuations around the average trend, which are more pronounced at smaller temperatures. They appear to be even larger than those reported in [4], but they are similar to the results of [29]. As for the friction coefficient, the dependence on deformation is particularly strong around the spherical shape. It reaches a kind of local maximum there, or perhaps at a slightly oblate shape. These features can be exhibited more clearly for parameterization in terms of $\epsilon$, for which reason we have made this choice here.

Many of these features point to the importance of shell effects, in particular the peak for friction around the spherical configuration, which is clearly visible for $T \approx 1-2 \mathrm{MeV}$ but which disappears at $T \approx 4-5 \mathrm{MeV}$. We like to elaborate on this statement by trying to split the friction coefficient up into a smooth and a fluctuating part. For this study we take the zero frequency limit.

Let us suppose for a moment that the matrix elements $\left|F_{j k}\right|^{2}$ considered as function of the single-particle energies have some smooth average component $\mathcal{F}^{2}\left(e, e^{\prime}\right)$ and the oscillating component can be neglected. In this case one may rewrite eq.(4.8) in the form

$$
\gamma(0) \approx-\hbar \pi \mathcal{F}^{2}(\mu, \mu) \int d \Omega \frac{\partial n(\Omega)}{\partial \Omega} g^{2}(\hbar \Omega)
$$

The $\varrho_{k}(\Omega)$ in $(4.8)$ are peaked functions with their maximum at $\hbar \Omega=\epsilon_{k}$ so that the sum of $\varrho_{k}$ over $k$ may be interpreted as the density $g(\hbar \Omega)$ of single-particle states. As usual the latter can be split into the smooth and oscillating components $g(e)=\tilde{g}(e)+\delta g(e)$ with $\tilde{g}(e)=\langle g(e)\rangle$ and $\langle\delta g(e)\rangle=0$ where the brackets stand for an averaging over the single-particle spectrum. Inserting this decomposition into (5.8), and noting that an integration performed with the bell-like function $\partial n(\Omega) / \partial \Omega$ can be understood like the average introduced above, we will get

$$
\gamma(0) \approx \hbar \pi\left[\bar{g}^{2}(\mu)+\left\langle\delta g^{2}(e)\right\rangle_{e=\mu}\right] \mathcal{F}^{2}(\mu, \mu)
$$

Assuming the oscillating component of the density to be periodic in the energy with some period $\hbar \Omega_{0}$ and amplitude $\delta g_{0}$, viz $\delta g(e)=\delta g_{0} \sin 2 \pi e / \hbar \Omega_{0}$, it is easy to convince oneselves that $\left\langle\delta g^{2}(e)\right\rangle=(1 / 2) \delta g_{0}^{2}$. The quantity $\delta g_{0}$ is determined by the magnitude of the shell correction. It is a smooth function of particle number ( see [31]) but still depends on deformation. It is maximal at that deformation where the shell structure is more pronounced. For the Woods-Saxon potential this happens to be so at the spherical shape. So it is due to the shell structure that the friction coefficient gains additional contributions around the sphere. For the case of the Woods-Saxon potential this specific feature seems to be responsible for the dip one sees in Fig.10 both for friction as well as for the inertia around $r_{12} \approx 0.5$, even at the somewhat larger temperatures of $3 \mathrm{MeV}$. It may be said, however, that such a behavior is not seen in computations performed with the model of 4 . There the change of the friction coefficient with $r_{12}$, for instance, resembles more the smooth one given by the wall formula.

\subsection{Collective relaxation times}

It has become customary to parameterize friction in terms of the ratio $\beta=\gamma / M$, although most of the interest has concentrated on friction alone; often the inertia was simply taken 
to be the reduced mass of the fissioning system. As we have seen above, for a microscopic theory both quantities will vary not only with the collective variable but with temperature as well. It may be expected, of course, that for the ratio the dependence on shape is much weaker than that of the individual quantities. This follows simply from the observation that any common, purely geometrical factor will drop out. In Fig.13 we present the results for $\beta$ obtained from those for inertia and friction discussed before. Indeed, this quantity is essentially constant over the whole deformation region, for all computations but $T=1 \mathrm{MeV}$, a case for which the fluctuations seen in our results are too big because of our neglecting pairing. However, there is a marked dependence on excitation: $\beta$ increases strongly with $T$. This is in clear distinction to the result one gets from applying the wall formula for friction and that of irrotational flow for the inertia. Interestingly enough, these macroscopic estimates lead to some $Q$-dependence, which in a sense is even bigger than suggested by the trend of our results. It can be said that the latter are very close to those obtained in the computations with a two-center shell model potential [⿴囗十].

Physically, the inverse of $\beta$ can be interpreted as the relaxation time $\left(\tau_{\text {kin }}=\mathrm{M} / \gamma\right)$ to the Maxwell distribution for collective motion. In full glory this feature can only be understood looking at the dynamics in collective phase space (see e.g. 16]). However, one may grasp its content recalling the local equation for average motion of the damped oscillator: $M \ddot{q}(t)+\gamma \dot{q}(t)+C q(t)=0$. From this equation it also becomes apparent that yet another relaxation time can be defined, namely $\tau_{\text {coll }}=\gamma /|C|$. For overdamped motion the latter is the only relevant one. Actually, such a situation is given for temperatures above $1-2 \mathrm{MeV}$.

Let us infer this feature comparing the macroscopic relaxation times with the microscopic $\tau$ found in sect.4.3. There we got values in the range between $\tau=0.3$ and $0.1 \hbar / \mathrm{MeV}$ for temperatures between 2 and $5 \mathrm{MeV}$, discarding the case of $\mathrm{T}=1 \mathrm{MeV}$ for the moment. Taking for the $\beta$ of Fig.13 typical values like 2 and $8 \mathrm{MeV} / \hbar$ for $T=2$ and $5 \mathrm{MeV}$, respectively, we recognize the $\tau_{\text {kin }}$ to be comparable or only slightly larger than $\tau$. This means that in this range of temperatures the friction force is so strong that it leads to an instant damping or disappearance of the kinetic energy. This is not in contradiction to the basic assumption behind the quasi-static picture. To justify the latter all one needs to have is the motion in $Q$ to be slow compared to that for the intrinsic degrees of freedom. For this question it is the $\tau_{\text {coll }}$ which becomes relevant. Its value can be estimated with the help of Figs. 6 and 10. Let us concentrate on deformations for which the local stiffness does not get zero. Since for $T \geq 2 \mathrm{MeV}$ shell effects are not important anymore we may estimate $C$ as the one of the liquid drop energy. The right part of Fig.6. tells us the $\left|C_{L D M}\right|$ to be of the order of $150 \mathrm{MeV}$ for $r_{12}$ larger than about 0.6. In this range the value of $\gamma$ is about $800 \hbar$, as seen from Fig. 10 (for $T=2 \mathrm{MeV}$; it is even bigger for larger temperatures). For $\tau_{\text {coll }}$ this implies values of the order of $5 \hbar / \mathrm{MeV}$, which are larger than $\tau$ by more than one order of magnitude.

\section{$6 \quad$ Summary and conclusions}

In this paper we have applied the single particle model of [3 to describe large scale motion at finite excitations. To this end this model had to be modified to include effects of collisional damping and it had to be adapted to the formulation of collective motion in the spirit of the locally harmonic approximation (for a review see [16]). Numerical computations have been performed for the transport coefficients of average motion along 
a fission path. The latter was identified by the valley in the potential landscape obtained for the liquid drop model. It was argued that for the range of temperatures considered in the present study this path may be expected to represent fairly well the actual situation. First of all, because of the evidence one has from static considerations that Cassini ovaloids describe well the shapes of the fissioning nucleus. Secondly, for the large damping one expects to be given, the system will be creeping down the collective potential and thus will stay close to the line of steepest descent.

For the transport coefficients values were found which are in accord with previous studies, in particular with the ones of 4 . This is especially so for their dependence with temperature, and to lesser extent for their variation with the nuclear shape. For instance, it turns out that typical effects of single particle motion become more apparent here than they did in [4], like there are fluctuations of both friction and inertia with the collective variable. To large amount they disappear when building ratios like for the $\beta=\gamma / M$ or the $\eta=\gamma /(2 \sqrt{M|C|}$, two quantities which are commonly used to parameterize collective dynamics.

A new development has been achieved with respect to comparisons with macroscopic models, like that of irrotational flow for the inertia and that of wall friction. As one knows, both are calculated for sharp density distributions which may differ considerably from those which correspond to the nucleons' densities in the shell model. A transformation was suggested which allows one to connect both density distributions and thus enables one to connect the transport coefficients accordingly. This transformation was found by applying a Strutinsky smoothing procedure to evaluate the average static value of the relevant one-body operator $\hat{F}\left(\hat{x}_{i}, \hat{p}_{i}, Q_{0}\right)$. It is this operator which by a self consistency argument is related to the deformation of the mean field.

Finally, we briefly like to turn to comparisons with experimental findings. First of all, we may mention that the values for $\beta$ shown above concur with the range suggested by fission experiments [32]. Two other relevant parameters are the $\varpi=\sqrt{|C| / M}$ and the $\eta$ mentioned above, which for instance appear in Kramers' famous formula for the decay rate of a one-dimensional fission model: $\mathrm{R}_{\mathrm{K}}=\left(\sqrt{1+\eta_{s}}-\eta_{s}\right)\left(\varpi_{m} / 2 \pi\right) \exp (-B / T)$. Here, $B$ measures the barrier height, $\varpi_{m}$ determines the vibrational frequency in the potential minimum and $\eta_{s}$ is to be evaluated at the saddle. The latter two quantities are shown in Figs.14 and 15, respectively, for both points. They have been calculated by averaging the $Q$-dependent transport coefficients in the neighborhood of the minimum and the barrier, where both are those of the $T$-dependent potential energy. These results agree very well with those already shown in [4]. It is remarkable that $\varpi$ does not change much with $T$, neither at the minimum nor at the barrier. Conversely, like the $\beta$ shown before, the $\eta$ too definitely increases with temperature. This behavior is in at least qualitative agreement with the one found in [33] and the numbers for $\eta$ lie in the range of values deduced only recently in 34 from experimental evidence. It is true, of course, that for more quantitative analyses one would have to consider both the effects of pairing as well as of angular momentum, not to mention the fact that there is some freedom [16] in adjusting the two parameters which define collisional damping. However, there is probably little doubt about the temperature dependence of our coefficients, say above $T=1.5-2 \mathrm{MeV}$. This marked change with $T$ is in clear distinction to the macroscopic models mentioned. In the light of this feature agreements between experimental findings and theoretical descriptions appear somewhat questionable if they are only based on these macroscopic models [7], [8]. Likewise the somewhat peculiar behavior of $\beta$ with $r_{12}$ suggested in [9] 
is not confirmed by our results (see also [4]). It is perhaps fair to say that some of the difficulties one still encounters at present when comparing theory with experiment is due to the high complexity of the problem itself as well that of the analysis of the data. To underline this statement, we just like to take up a point raised in [10], namely that common statistical codes evaluate the fission decay rate not by the transition state result, to which Kramers' formula reduces to for $\eta_{s} \rightarrow 0$, but by that of the "statistical model" where $\varpi_{m}$ is replaced by $T$. The difference between these two variants can easily be inferred from Fig.14.

Acknowledgments. The authors want to thank the Deutsche Forschungsgemeinschat for financial support. Two of us (F.A.I and V.V.P.) would like to thank the Physics Department of the TUM for the hospitality extended to them during their stay in Garching.

\section{A Intrinsic response function}

The computation of the intrinsic response function is somewhat involved. Due to the frequency dependence both of Fermi distribution $n(\omega)$ and width $\Gamma(\omega)$ the integral in (4.2) can not be calculated analytically. The numerical integration is rather time consuming since $\varrho_{k}(\omega)$ are sharply peaked functions which width varies by two order of magnitude depending on the values of $e_{k}$. Fortunately the integration in (4.2) can be carried out by means of residues theorem closing the integration limit in the lower half plane. For this one needs to find the poles and residues of all the terms in the integrand of (4.2).

Let us first look for the poles of $\mathcal{G}_{k}(\omega+i \epsilon)$. Substituting (4.3), (4.4) into (4.6) and introducing notation $\Delta \equiv \sqrt{c^{2}+\pi^{2} T^{2}}, \mu^{ \pm}=\mu \pm i \Delta$ (4.6) can be brought to the form

$$
\begin{gathered}
\mathcal{G}_{k}(\omega+i \epsilon)=\left(\hbar \omega-\mu^{+}\right)\left(\hbar \omega-\mu^{-}\right) \times \\
\left\{\left(\hbar \omega-e_{k}\right)\left(\hbar \omega-\mu^{+}\right)\left(\hbar \omega-\mu^{-}\right)+\frac{\left[\hbar \omega-\mu^{+}+i \Delta\right]}{2 \Gamma_{0} \Delta / c^{4}}+\frac{i\left[\left(\hbar \omega-\mu^{+}\right)\left(\hbar \omega-\mu^{-}\right)-c^{2}\right]}{2 \Gamma_{0} / c^{2}}\right\}^{-1}
\end{gathered}
$$

It is not difficult to note that the last terms in square brackets cancel with each other and both numerator and denominator can be divided by $\hbar \omega-\mu^{+}$. In this way $\mathcal{G}_{k}(\omega+i \epsilon)$ becomes

$$
\mathcal{G}_{k}(\omega+i \epsilon)=\frac{\hbar \omega-\mu^{-}}{\left(\hbar \omega-\hbar \omega_{k}^{+}\right)\left(\hbar \omega-\hbar \omega_{k}^{-}\right)}=\frac{1}{\hbar^{2}\left(\omega_{k}^{+}-\omega_{k}^{-}\right)}\left[\frac{\hbar \omega_{k}^{+}-\mu^{-}}{\omega-\omega_{k}^{+}}+\frac{\mu^{-} \hbar \omega_{k}^{-}}{\omega-\omega_{k}^{-}}\right]
$$

where $\hbar \omega_{k}^{ \pm}$are solutions of the equation

$$
\left(\hbar \omega-\hbar \omega_{k}^{+}\right)\left(\hbar \omega-\hbar \omega_{k}^{-}\right) \equiv\left(\hbar \omega-e_{k}+i \frac{c^{2}}{2 \Gamma_{0}}\right)\left(\hbar \omega-\mu^{-}\right)+\frac{c^{4}}{2 \Gamma_{0} \Delta}=0
$$

namely

$$
\hbar \omega_{k}^{ \pm}=\frac{1}{2}\left\{e_{k}+\mu^{-}-i \frac{c^{2}}{2 \Gamma_{0}} \pm\left[\left(e_{k}-\mu^{-}-i \frac{c^{2}}{2 \Gamma_{0}}\right)^{2}-\frac{2 c^{4}}{\Gamma_{0} \Delta}\right]^{1 / 2}\right\}
$$

The both poles of $\mathcal{G}_{k}(\omega+i \epsilon)$ lie in the lower half-plane. The residues of $\mathcal{G}_{k}(\omega+i \epsilon)$ are simple functions of $\hbar \omega_{k}^{ \pm}$and $\mu^{-}$as it is seen from (A.2). From the definition (4.6) it is easy to seen that poles and residues of $\mathcal{G}_{k}(\omega-i \epsilon)$ are complex conjugated to that of 
$\mathcal{G}_{k}(\omega+i \epsilon)$. The pole representation for $\varrho_{k}(\omega)$ is easily obtained from (A.2). Besides the poles of $\mathcal{G}_{k}^{ \pm}(\omega \pm i \epsilon)$ and $\varrho_{k}(\omega)$ one should account also for the poles of Fermi function $n(\omega)$ in the plane of complex $\omega$ (so-called Matzubara frequencies)

$$
\hbar \omega_{n}=\mu \pm i \pi T(2 n+1), \quad n=0,1,2, \ldots
$$

In principle, the sum extends over infinite many terms, but in practice the summation in (4.2) is cut at such frequencies $\omega_{n}$ which contribute less than determined by the desired accuracy.

\section{References}

[1] V.M.Strutinsky, N.Ya.Lashchenko and N.A.Popov, Nucl.Phys. 46, 639 (1963)

[2] V.S. Stavinsky, N.S. Rabotnov and A.A. Seregin, Yad.Fiz. 7, 1051 (1968)

[3] V.V. Pashkevich, Nucl. Phys. A169, 275 (1971)

[4] S. Yamaji, F.A. Ivanyuk and H. Hofmann, Nucl.Phys. A, in press

[5] A. Bohr and B. Mottelson. Nuclear Structure, vol. 2, (Benjamin, London, 1975)

[6] R. Samhammer, Doctoral Thesis, Technical University Munich (1988) and R. Samhammer, H. Hofmann and S. Yamaji, Nucl. Phys. A503, (1989) 404

[7] T. Wada, N. Carjan and A. Abe, Nucl.Phys. A538, 283c (1992); Phys.Rev. Lett. 70, 3538 (1993)

[8] K. Pomorski, J. Bartel, J. Richert and K. Dietrich, Nucl. Phys. A605, 87 (1996)

[9] I.I. Gontchar and P. Fröbrich, Physics of Atomic Nuclei. 57, 1181 (1994)

[10] M. Thoennessen, Nucl. Phys. A 599, 1 (1996)

[11] J. Damgaard, H.C. Pauli, V.V. Pashkevich and V.M. Strutinsky, Nucl.Phys. A135, $432(1969)$

[12] V.M. Strutinsky, Nucl.Phys. A95, 420 (1967); A122, 1 (1968)

[13] M. Brack, J. Damgaard, H.C. Pauli, A.S. Jensen, V.M. Strutinsky and C.Y. Wong, Rev.Mod.Phys. 44, 420 (1972)

[14] C. Guet, E. Strumberger and M. Brack. Phys.Lett. 205 B, 427 (1988)

[15] P.J. Siemens and A.S. Jensen, "Elements of Nuclei: Many-Body Physics with the Strong Interaction", Addison and Wesley, 1987

[16] H. Hofmann, Phys. Reports, in press

[17] D. Kiderlen, H. Hofmann and F.A. Ivanyuk, Nucl. Phys. A550, 473 (1992)

[18] H.Hofmann, F.A.Ivanyuk and S.Yamaji, Nucl. Phys. A598, 187 (1996) 
[19] H. Hofmann, Phys.Lett. 61 B, 423 (1976)

[20] H. Hofmann, R. Samhammer and S. Yamaji, Phys. Lett. 229 B, 309 (1989)

[21] W.J. Swiatecki, Nucl.Phys. A574, 233c (1994)

[22] H. Hofmann and F.A. Ivanyuk, Z.Phys. A, Hadrons and Nuclei. 344, 285 (1993)

[23] R. Alkofer, H. Hofmann and P.J. Siemens, Nucl. Phys. A476, 213 (1988)

[24] S. Yamaji, H. Hofmann and R. Samhammer, Nucl. Phys. A475, 487 (1988)

[25] A. S. Jensen, J. Leffers, H. Hofmann and P.J. Siemens, Physica Scripta. T5, 186 (1983)

[26] J. Blocki, Y. Boneh, J.R. Nix, J. Randrup, M. Robel, A.J. Sierk, W.J. Swiatecki. Ann. Phys. 113, 330 (1978)

[27] F.A. Ivanyuk, V.M. Kolomietz and A.G. Magner, Phys.Rev. C52, 678 (1995)

[28] K.T.R. Davis, A.J. Sierk and J.R. Nix, Phys.Rev. C13, 2385 (1976)

[29] F.A. Ivanyuk and K. Pomorski, Phys.Rev. C53, 1861 (1996)

[30] H. Hofmann, S. Yamaji and A.S. Jensen, Phys. Lett. 286 B, 1 (1992)

[31] F.A.Ivanyuk and V.M.Strutinsky, Z. Phys. A293, 337 (1979)

[32] D. Hilscher, I.I. Gontchar and H. Rossner, Physics of Atomic Nuclei. 57, 1187 (1994)

[33] D.J. Hofman, B.B. Back, I. Diószegi, C.P. Montoya, S. Schadmand, R.Varma, and P. Paul, Phys. Rev.Let. 72, 470 (1994); see also: P. Paul and M. Thoennessen, Ann.Rev.Part.Nucl.Sci. 44, 65 (1994)

[34] J.P. Lestone, Tours Symposium on Nuclear Physics II, pg 458-469, World Scientific Publishing Co. (1995) ISBN 981-02-2156 and preprint 1996; see also J.P. Lestone, Phys. Rev. Lett. 70 (1993) 2245 


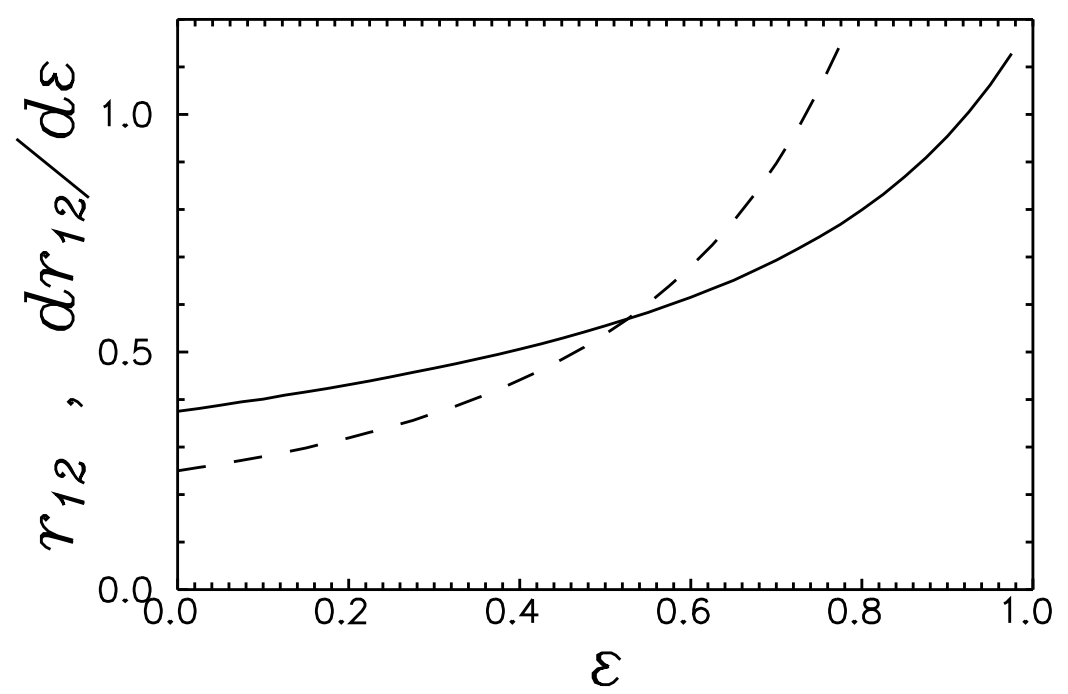

Figure 2: The relation between $r_{12}$ and $\epsilon$ (solid line); the derivative $d r_{12} / d \epsilon$ is shown by dashed line.

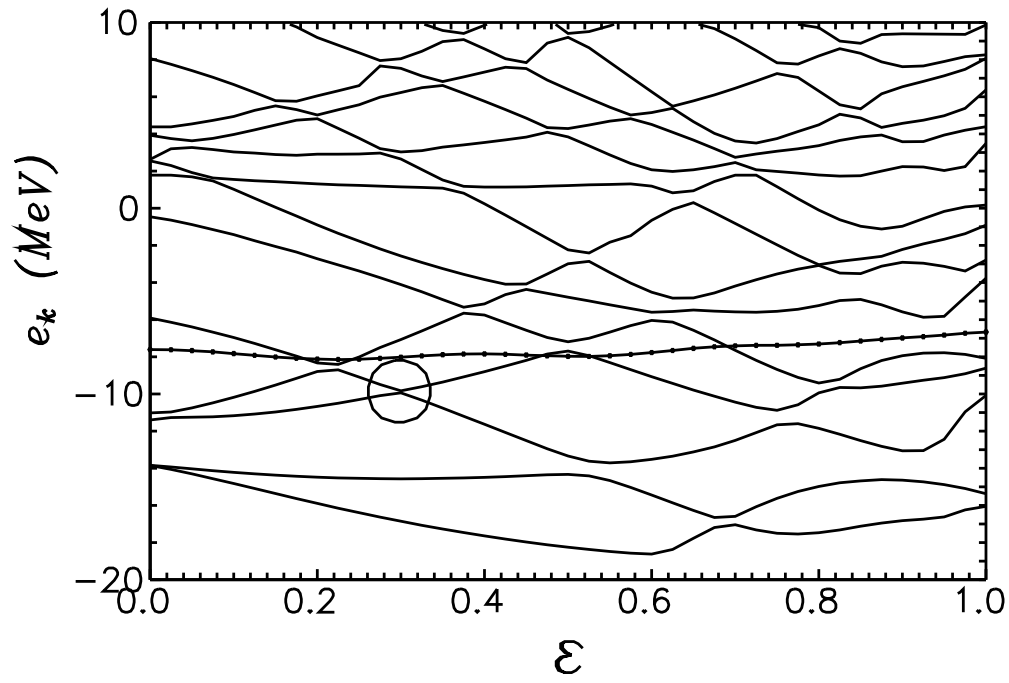

Figure 3: The energies $e_{k}$ of single-particle states (for fixed $z$-component of angular momentum and parity, $j_{z}^{\pi}=3 / 2^{-}$) as functions of $\epsilon$. The line with stars marks the position of the chemical potential computed for $T=1 \mathrm{MeV}$. The circle marks the pseudo crossing which at $\epsilon=0.3$ is closest to the chemical potential, see text. 

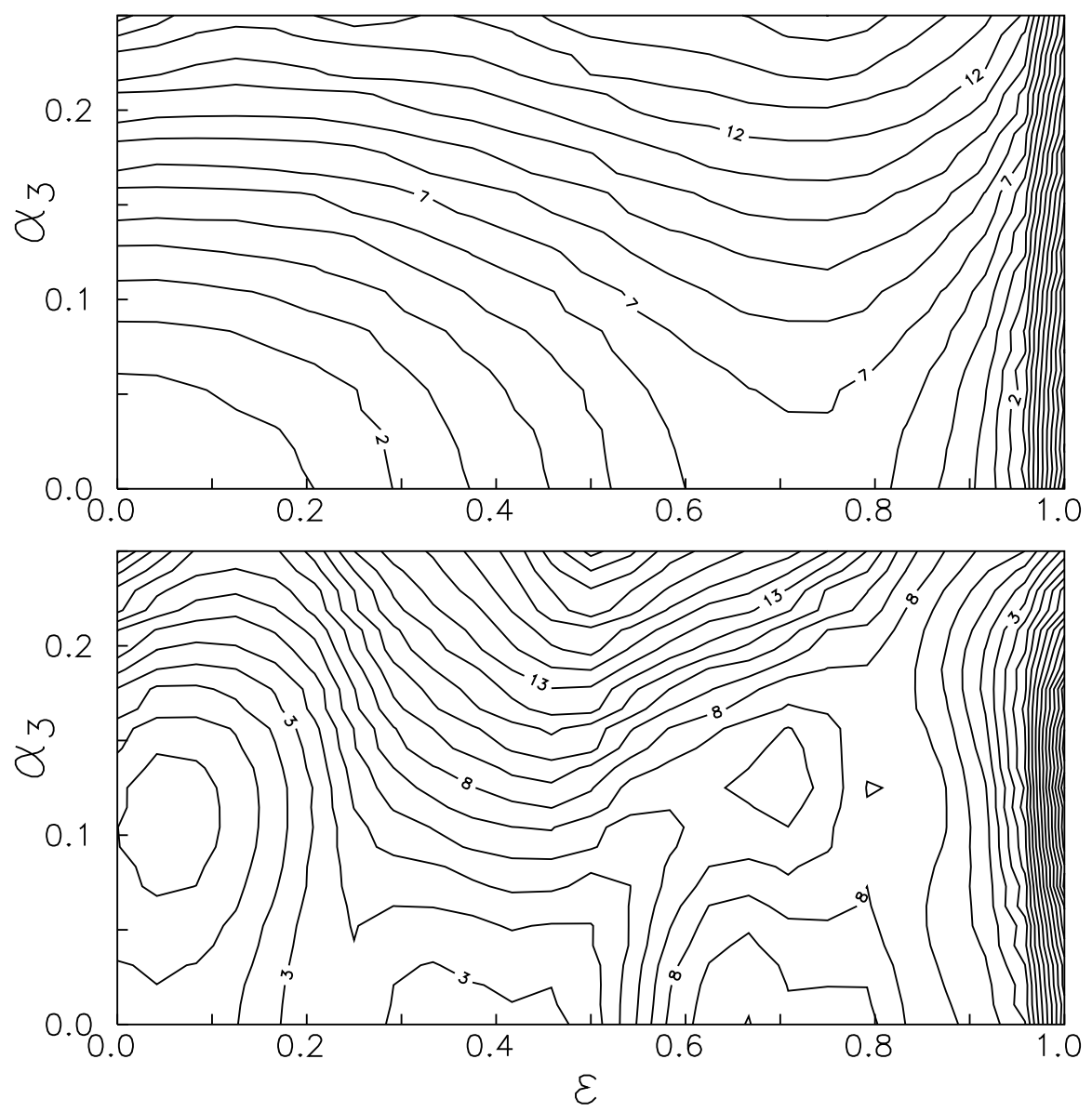

Figure 4: The liquid drop (top) and total (bottom) deformation energies of ${ }^{224} T h$ at $T=0$ as function of $\epsilon$ and $\alpha_{3}$.

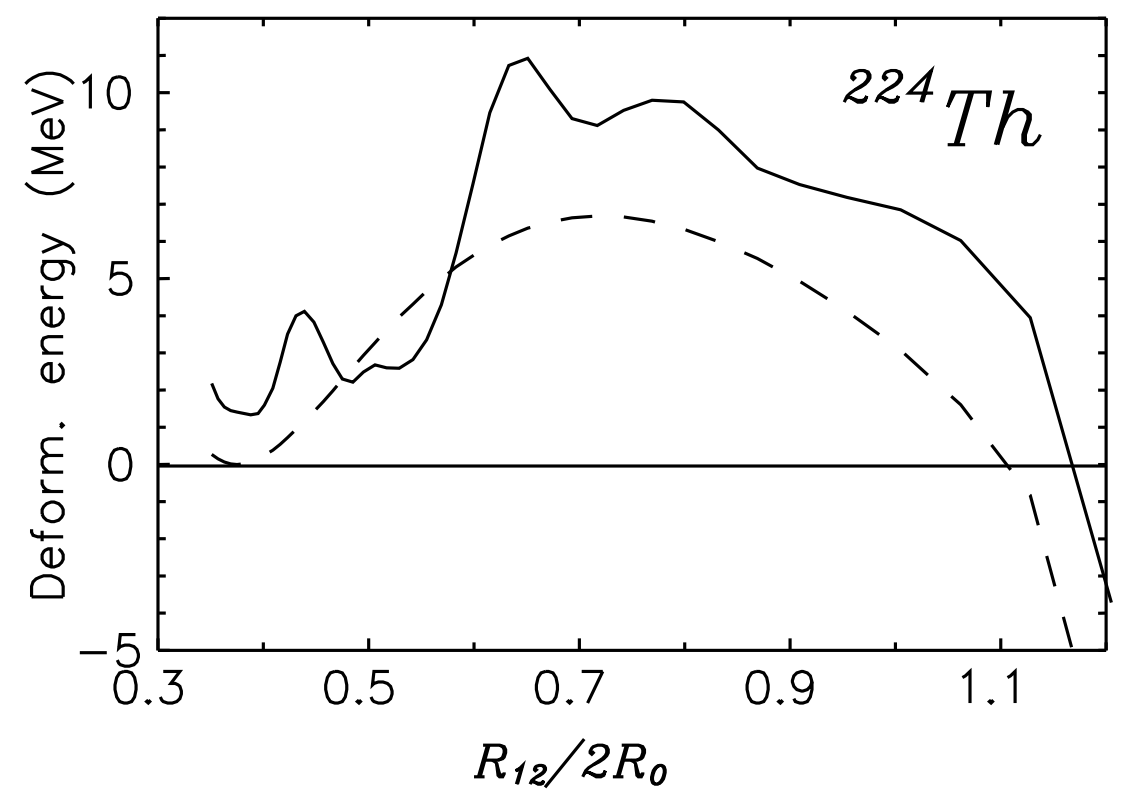

Figure 5: The total (solid) and liquid (dashed) drop components of the deformation energy along the liquid drop fission valley for ${ }^{224} \mathrm{Th}$. 

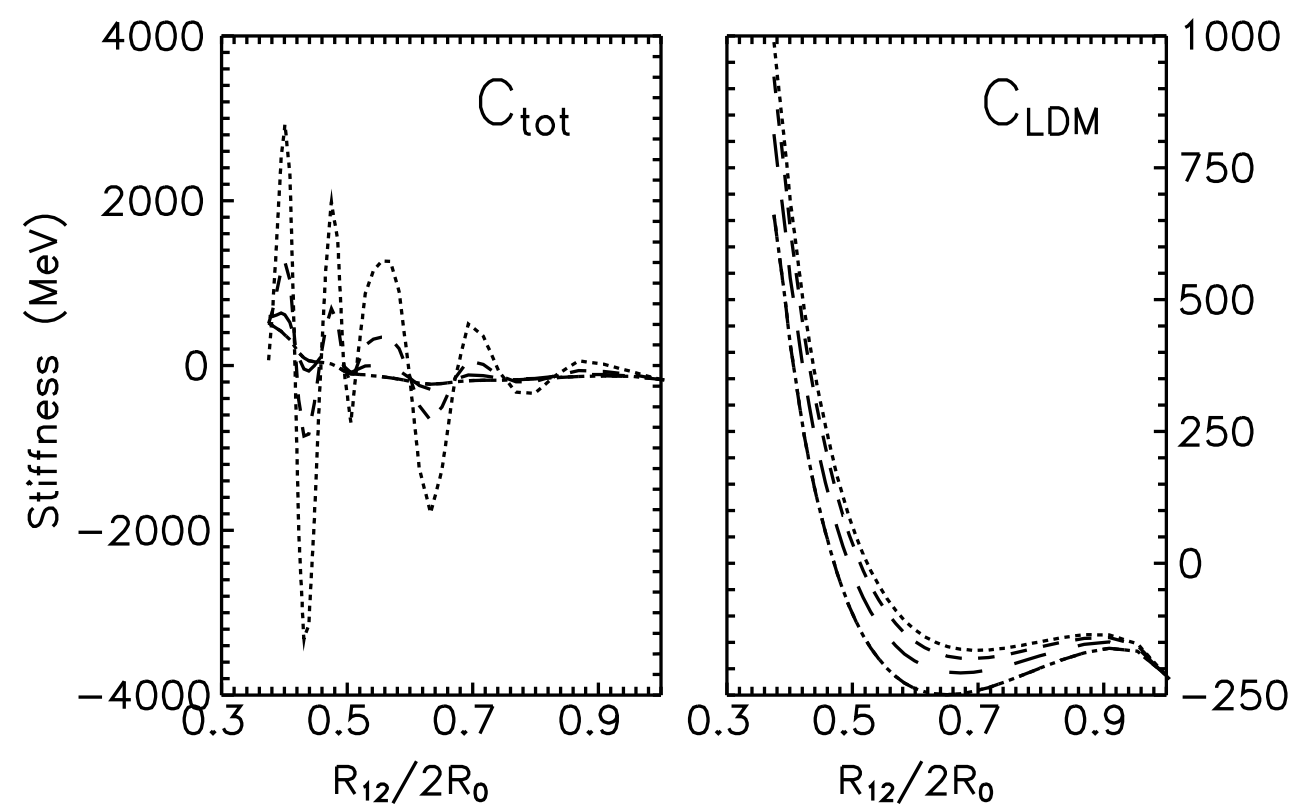

Figure 6: The zero frequency limit (3.8) for the stiffness of the total (left) and liquid drop (right) static energies. The dotted, short-dashed, long-dashed and dotted-dashed lines correspond to temperatures $T=1,2,3$ and $4 \mathrm{MeV}$.

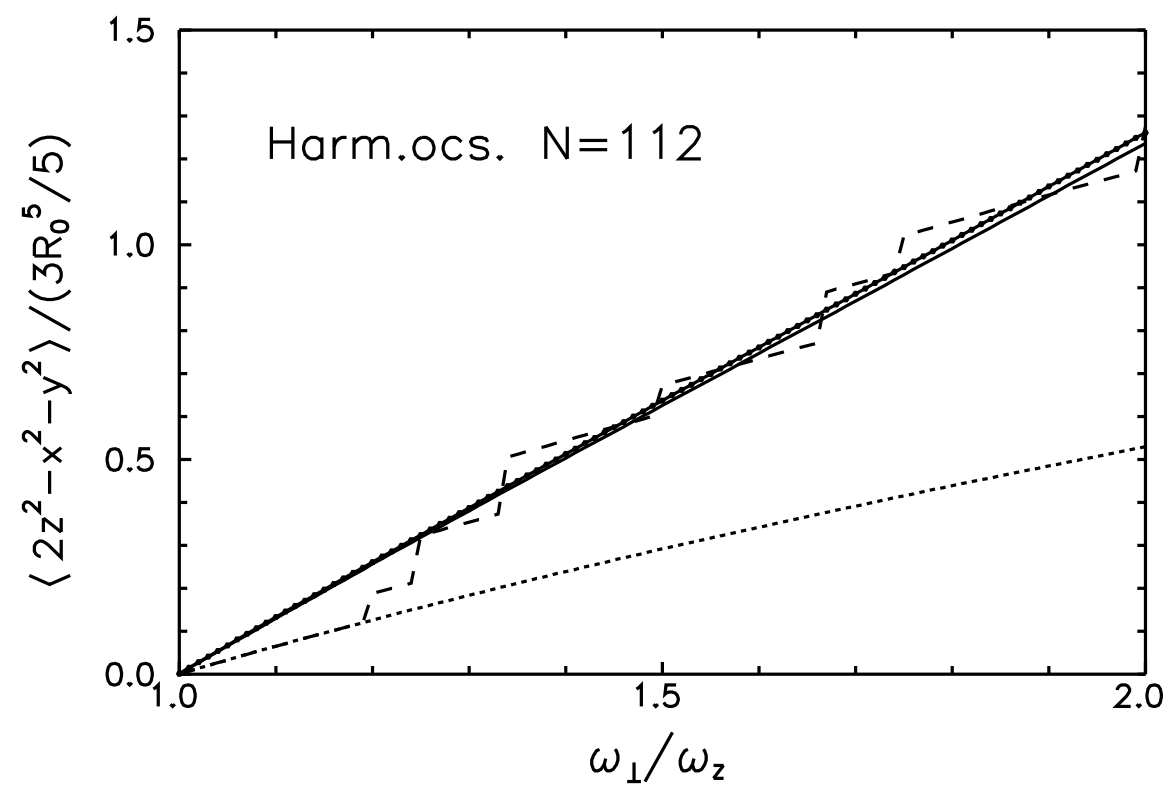

Figure 7: A demonstration of the problem of consistency between the deformation of potential and density, exemplified at the deformed oscillator; for details see text. 

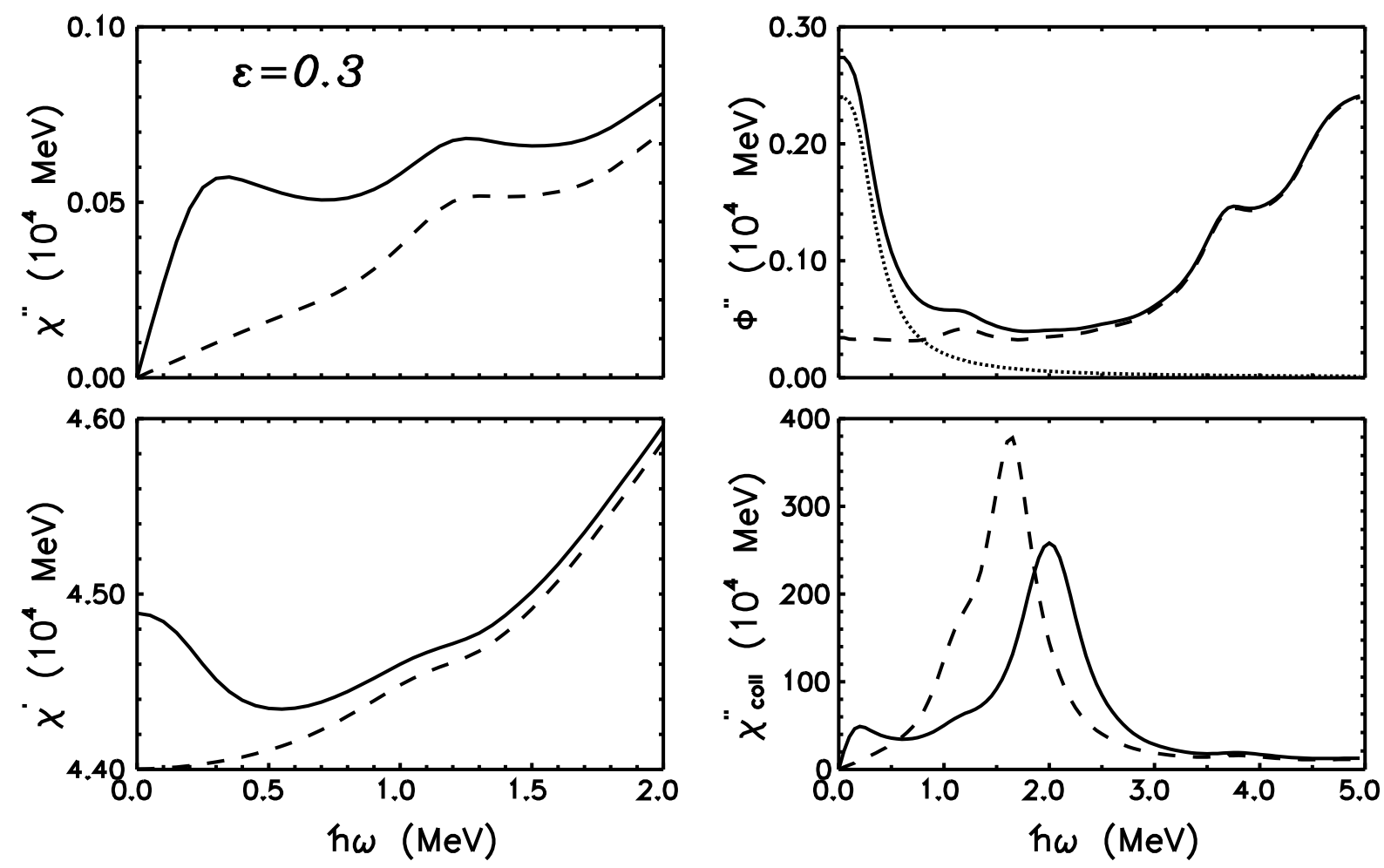

Figure 8: The heat pole contribution to the relaxation and response functions, calculated at $T=1 \mathrm{MeV}$.
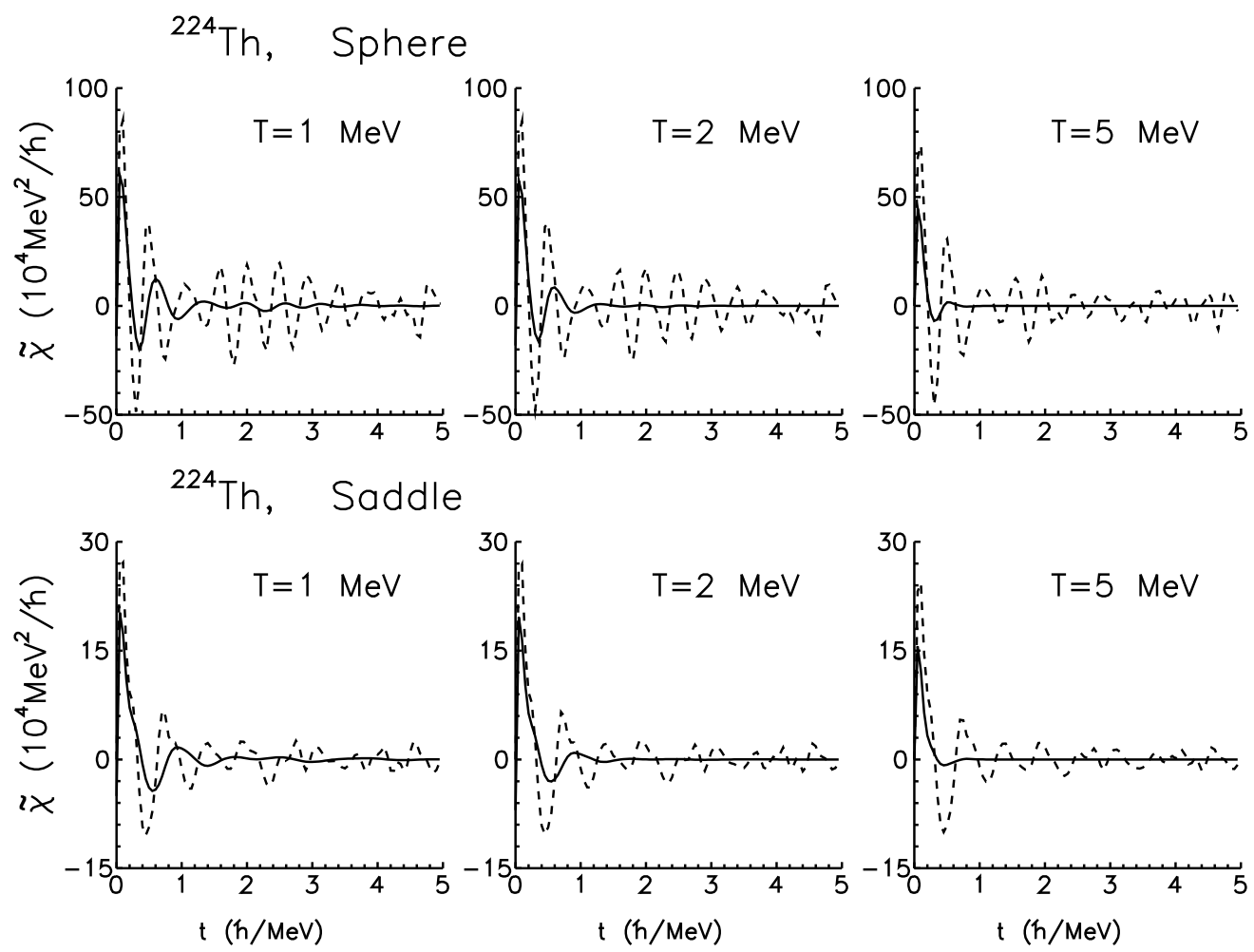

Figure 9: The time dependent function for the nucleonic response, calculated within the pure shell model (dashed curve) and for collisional damping (fully drawn line). 

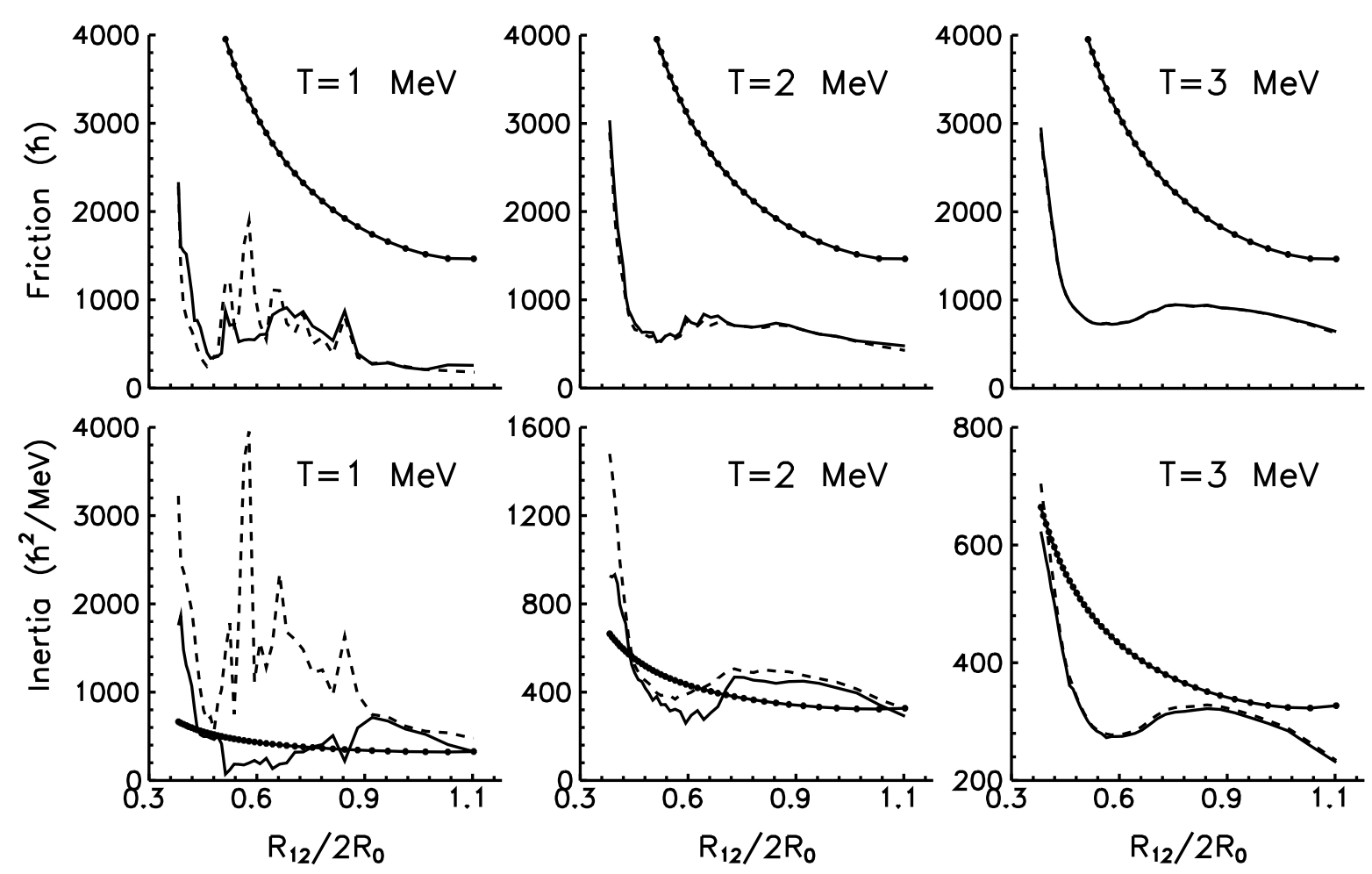

Figure 10: The friction coefficient and the mass parameter at finite frequency (solid lines) and for the approximation (3.17)-(3.18) (dashed lines).

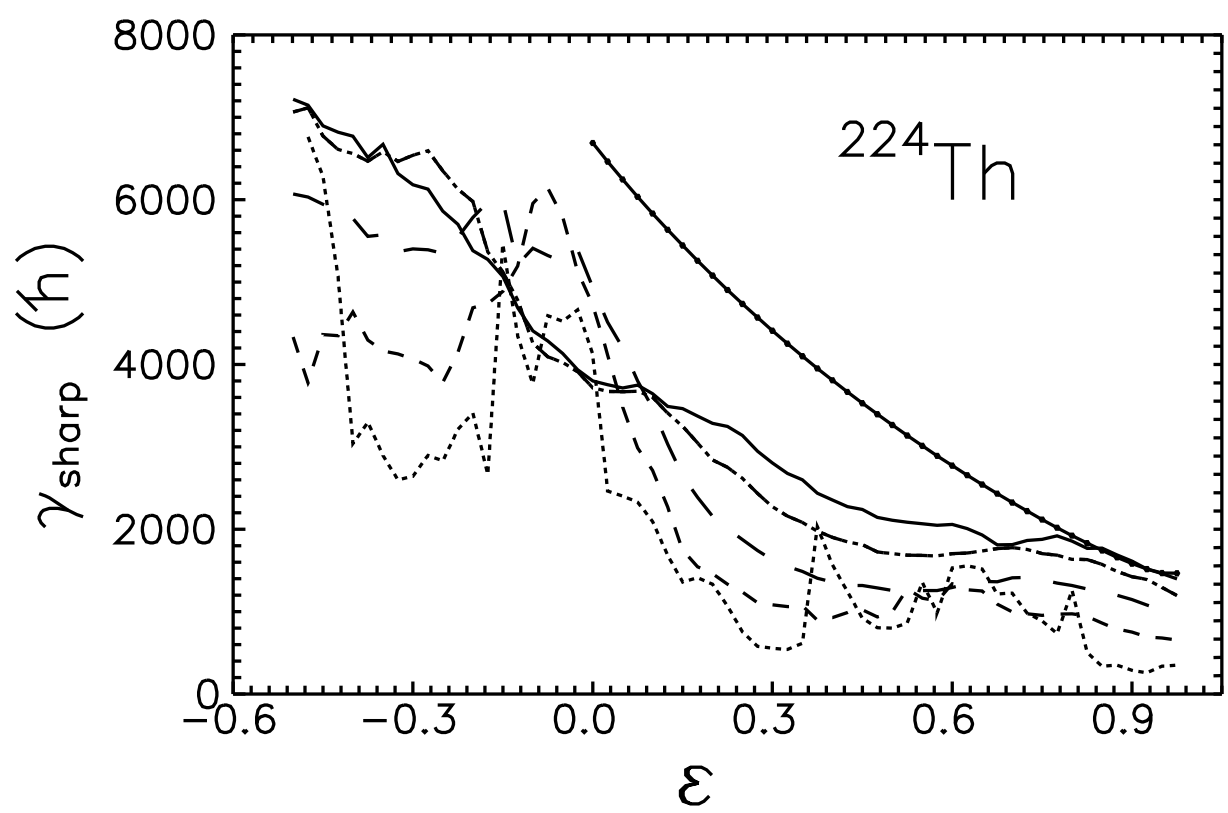

Figure 11: The deformation dependence of friction; the dotted, short-dashed, long-dashed, dotted-dashed and solid lines correspond to temperatures $T=1,2,3,4$ and $5 \mathrm{MeV}$. 


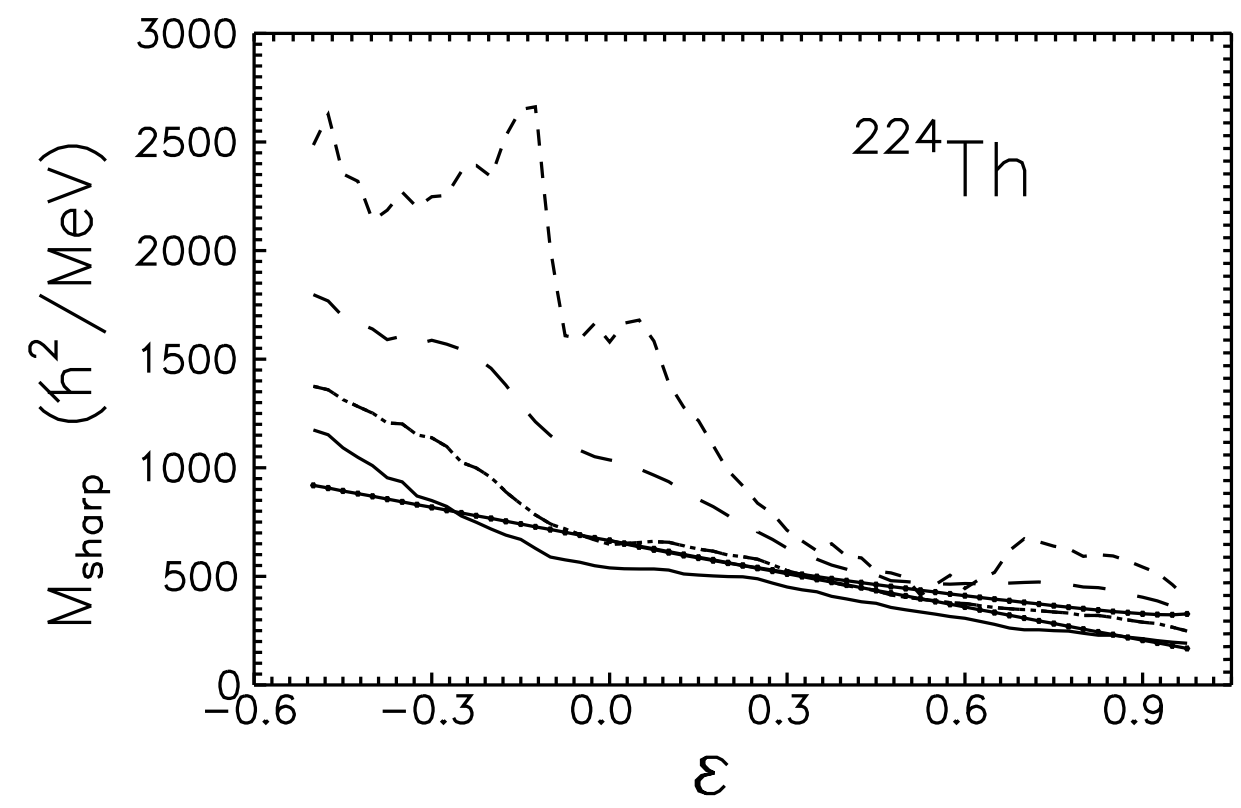

Figure 12: The deformation dependence of the mass parameter for temperatures $T=$ $1-5 \mathrm{MeV}$. Different temperatures are marked by the same lines as in Fig.11.

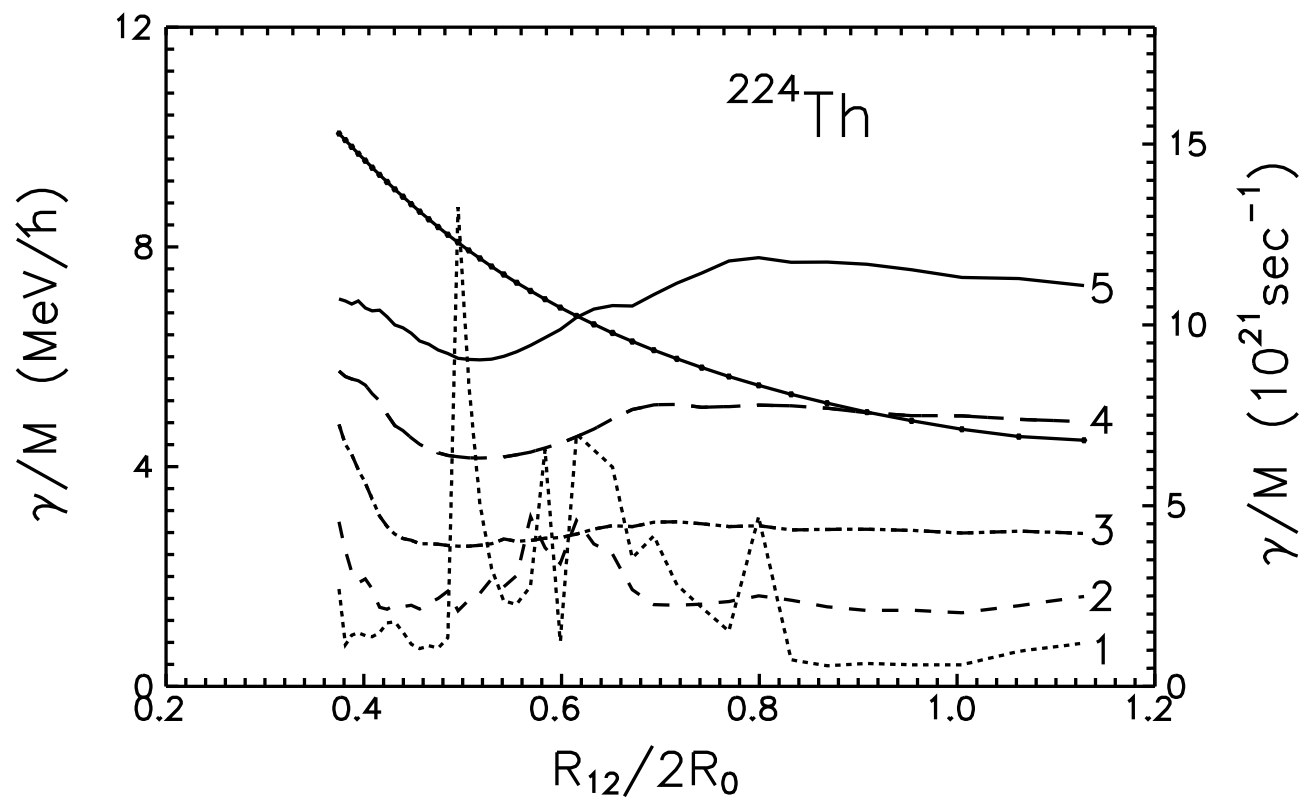

Figure 13: The inverse relaxation time $\beta=\gamma\left(\omega_{1}\right) / M\left(\omega_{1}\right)$ as function of deformation and temperature (indicated in the Figure). 


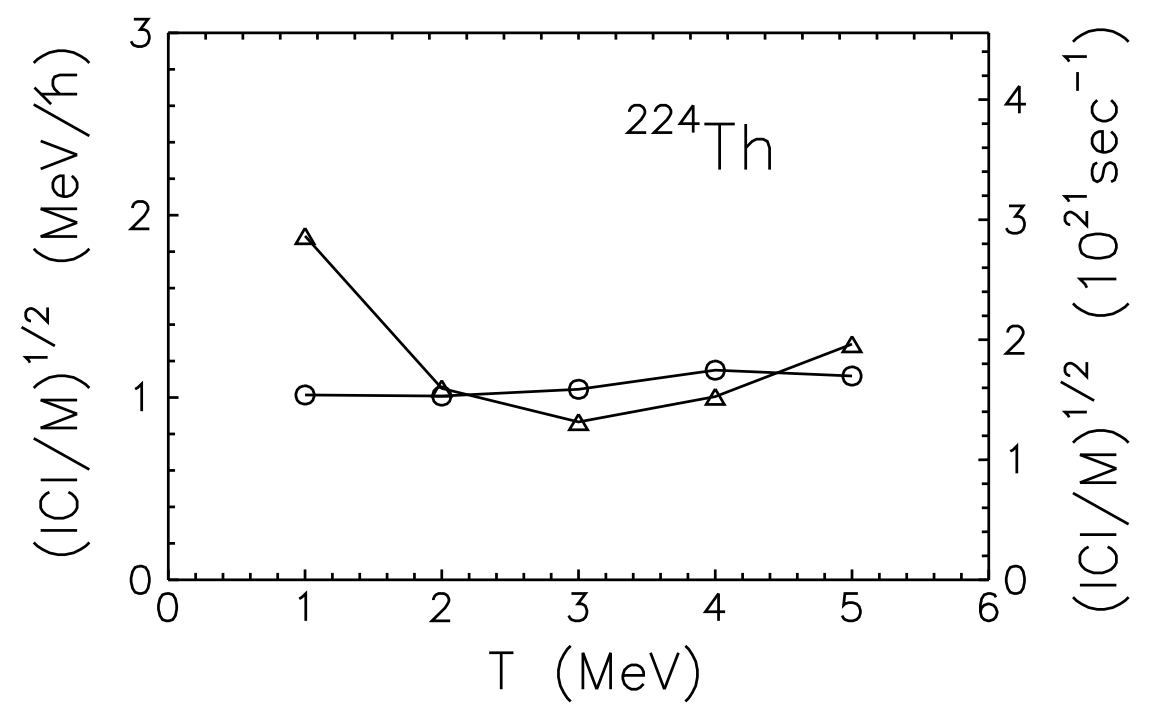

Figure 14: The vibrational frequency $\varpi=\sqrt{|C| / M}$ as function of temperature, calculated at the potential minimum (marked by circles) and at the saddle point (marked by triangles).

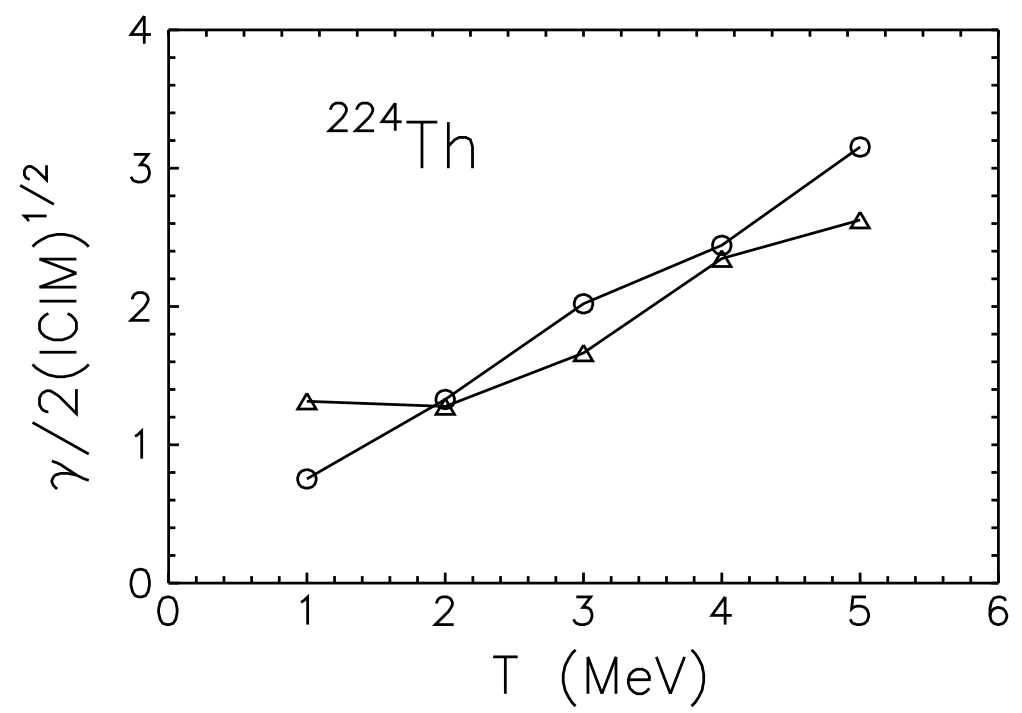

Figure 15: The dimensionless parameter $\eta=\gamma /(2 \sqrt{|C| M})$ as function of temperature, calculated at the potential minimum (marked by circles) and at the saddle point (marked by triangles). 